\title{
Structure and function revealed with submolecular resolution at the liquid-solid interface
}

\author{
Johannes A. A. W. Elemans and Steven De Feyter* \\ Received 30th June 2008, Accepted 30th October 2008 \\ First published as an Advance Article on the web 9th December 2008 \\ DOI: $10.1039 / b 811090 \mathrm{e}$
}

\begin{abstract}
The liquid-solid interface is a unique medium to support the self-assembly of molecules into surfaceconfined networks. Non-covalent interactions are key in forming these two-dimensional (2D) architectures, and a deep understanding is crucial for successful 2D crystal engineering. Scanning tunnelling microscopy is the tool of choice to reveal the structure and function of these patterns with subnanometre resolution. A recent success is the formation of 2D nanoporous molecular patterns and their host-guest chemistry. However, this is not the only functionality addressed by this review.

Surface-confined molecular architectures at the liquid-solid interface are also relevant in the field of molecular electronics. Furthermore, inducing and probing chemical reactivity at the single-molecule level at the liquid-solid interface might turn out to be one of the most exciting developments.
\end{abstract}

\section{Introduction}

Monolayers at liquid-solid interfaces play a very important role in a number of industrially relevant processes (e.g. lubrication) and are of intense academic interest, not least because of the possibilities in nanostructuring and functionalising surfaces. The structure and dynamics of such monolayers can be followed with a variety of tools such as neutron diffraction and incoherent neutron scattering. ${ }^{1}$ The invention of scanning probe microscopes has literally opened our eyes and revealed in real space the often complex structure and dynamics of molecular patterns. In

Department of Chemistry, Division of Molecular and Nano Materials, and INPAC-Institute for Nanoscale Physics and Chemistry, Katholieke Universiteit Leuven, Celestijnenlaan 200-F, 3001 Leuven, Belgium. E-mail: Steven.DeFeyter@chem.kuleuven.be; Fax: (+32) 16-327-990 particular, scanning tunnelling microscopy (STM) has proven to be an excellent tool for probing these molecular layers in detail. ${ }^{2}$

In this review, we will not deal with the immensely popular class of self-assembled monolayers of, for instance, alkyl thiols on gold. ${ }^{3}$ The molecular systems discussed here are only weakly physisorbed at the liquid-solid interface. ${ }^{4}$ In addition to important enthalpic contributions (molecule-molecule and moleculesubstrate interactions), the self-assembly of rather rigid solutes at the liquid-solid interface displacing small physisorbed solvent molecules is entropically favoured. The substrate is typically highly oriented pyrolytic graphite (HOPG) or Au(111). These atomically flat conductive surfaces are ideal for revealing molecular layer structure and properties by STM. Often only monolayers (e.g. not clusters or multilayers) are revealed. Successful STM imaging with submolecular resolution requires molecules to remain laterally immobilised during the time it takes the STM tip to scan the area (from several seconds to several

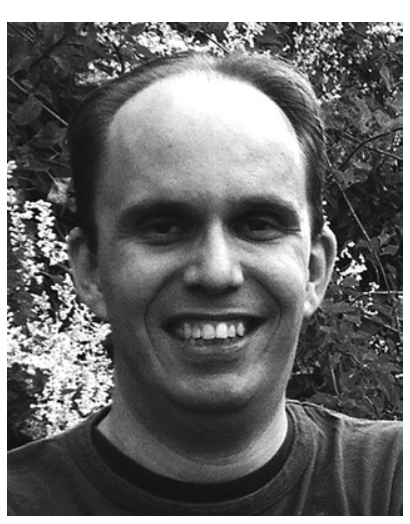

Hans Elemans
Hans Elemans completed his Ph.D. in 2001 with Roeland Nolte at the Radboud University Nijmegen (The Netherlands), where he then started investigating the self-organisation and reactivity of molecules at solidl liquid interfaces with STM. For this work he was awarded 'Veni' and 'Vidi' innovative research grants in 2004 and 2008. In 2008 he moved for one year to the group of Prof. Steven De Feyter at K.U.Leuven (Belgium) to work on various aspects of scanning probe microscopy. His scientific interests are at the interface of chemistry and physics, in particular in the areas of catalysis and self-assembled architectures.

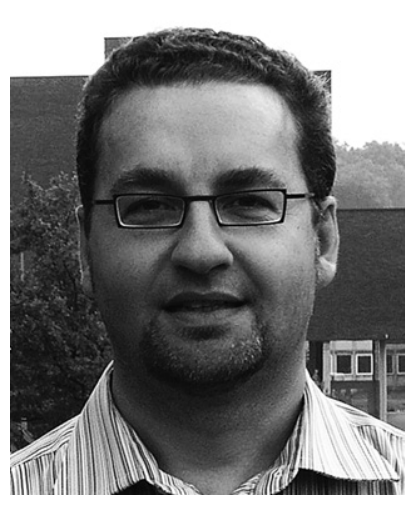

Steven De Feyter
Steven De Feyter (Kortrijk, Belgium, 1971) is professor at the Katholieke Universiteit Leuven (K.U.Leuven) in Belgium. After starting up scanning tunnelling microscopy during his PhD in the group of Prof. Frans C. De Schryver at K.U.Leuven he moved for a postdoctoral position to the group of Prof. Ahmed Zewail (California Institute of Technology, Pasadena) where he was involved in ultrafast organic femtochemistry. He rejoined the Leuven group in 1999. His current interests include the study of supramolecular chemistry and self-assembly phenomena at surfaces with scanning probe methods with a focus on liquid-solid interfaces. 


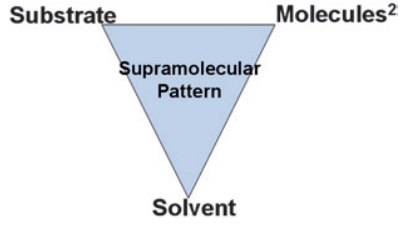

Scheme 1 The outcome of the molecular self-assembly process at a liquid-solid interface is the result of a complex interplay between molecule-molecule ("molecules"), molecule-solvent, moleculesubstrate, and solvent-substrate interactions.

minutes). Under physisorption conditions, at room temperature, most low-molecular weight molecules are too mobile to be visualised at the liquid-solid interface, except if they are trapped in or are part of a two-dimensional (crystalline) matrix. Multilayers are also observed less frequently than monolayers. Due to the exponential distance dependence of the tunnelling current, the distance between the tip and substrate is very small (on the order of a few Angstroms only). Given that subsequent molecular layers are often less stable or less ordered, they are easily 'removed' by the STM tip revealing only the first adsorbed layer.

The vast majority of these studies deal with the structural aspects of these monolayers, which one could call two-dimensional (2D) crystal engineering. ${ }^{5}$ Though such studies are absolutely necessary as they bring insight in the complexity of the interplay between molecules, substrate and solvent (Scheme 1), the next most important level is functionality. ${ }^{6}$ Key challenges are the identification of possible functionalities and the design and construction of these functional surfaces, based upon the self-assembly of weakly adsorbed molecules. This is what this review is about: structure and function at the liquid-solid interface, focussing on supramolecular host-guest networks and dynamics; and molecular electronics and reactivity, monitored or even induced by STM.

\subsection{Non-covalent interactions between molecules at liquid-solid interfaces}

In analogy to the situation in $3 \mathrm{D}$ crystals, the ordering of molecules within a $2 \mathrm{D}$ crystalline layer at a liquid-solid interface

A

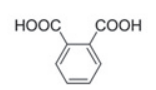

phthalic acid

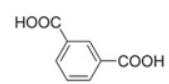

isophthalic acid (ISA)

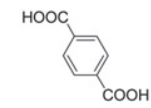

terephthalic acid (TTA)
B

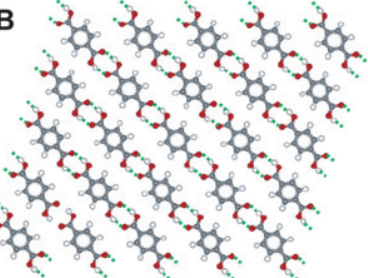

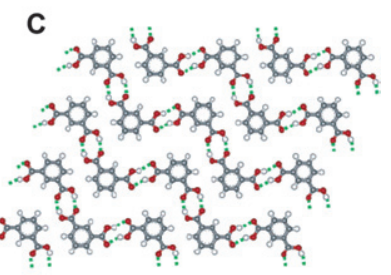

Fig. 1 (A) Molecular structures of phthalic acid, isophthalic acid (ISA) and terephthalic acid (TTA). (B) Arrangement of TTA, and (C) ISA at the interface of graphite and 1-heptanoic acid. (Reproduced with permission from ref. 7. Copyright 2004 American Chemical Society). is generally governed by non-covalent interactions. Amongst those, hydrogen bonding and metal-ligand coordination are frequently encountered motifs that determine the self-assembly of molecules, because they are relatively strong and highly directional. The role that hydrogen bonding can play in $2 \mathrm{D}$ crystallisation processes is perfectly illustrated by the behaviour of phthalic acid derivatives. ${ }^{7}$ These simple molecules consist of a benzene ring to which two carboxylic acid groups are attached. In the case of 1,2-benzenedicarboxylic acid (phthalic acid), no monolayers are formed at the liquid-solid interface because, due to steric hindrance, the carboxylic acid groups are not coplanar and therefore the molecule is not flat. In addition, it is conceptually impossible for this molecule to form extended arrays based upon hydrogen bonding. However, the 1,3-isomer (isophthalic acid, ISA) and the 1,4-isomer (terephthalic acid, TTA) form densely packed monolayers with a structure dominated by hydrogen bonding interactions. Encoded by their substitution pattern, TTA self-assembles into linear arrays, while ISA is organised in zig-zag patterns (Fig. 1).

In the case of metal-ligand coordination, transition metal centres are used to connect two or more organic ligands by means of highly specific and directional interactions. By making use of a library of available metal-ligand combinations, many parameters of the resulting architectures, such as binding stoichiometry, angle, strength, and reversibility, can be controlled and predicted. Most 2D metal-ligand arrays at a surface have been constructed under ultrahigh vacuum (UHV) conditions. ${ }^{8}$ However, examples of such arrays at a liquid-solid interface,
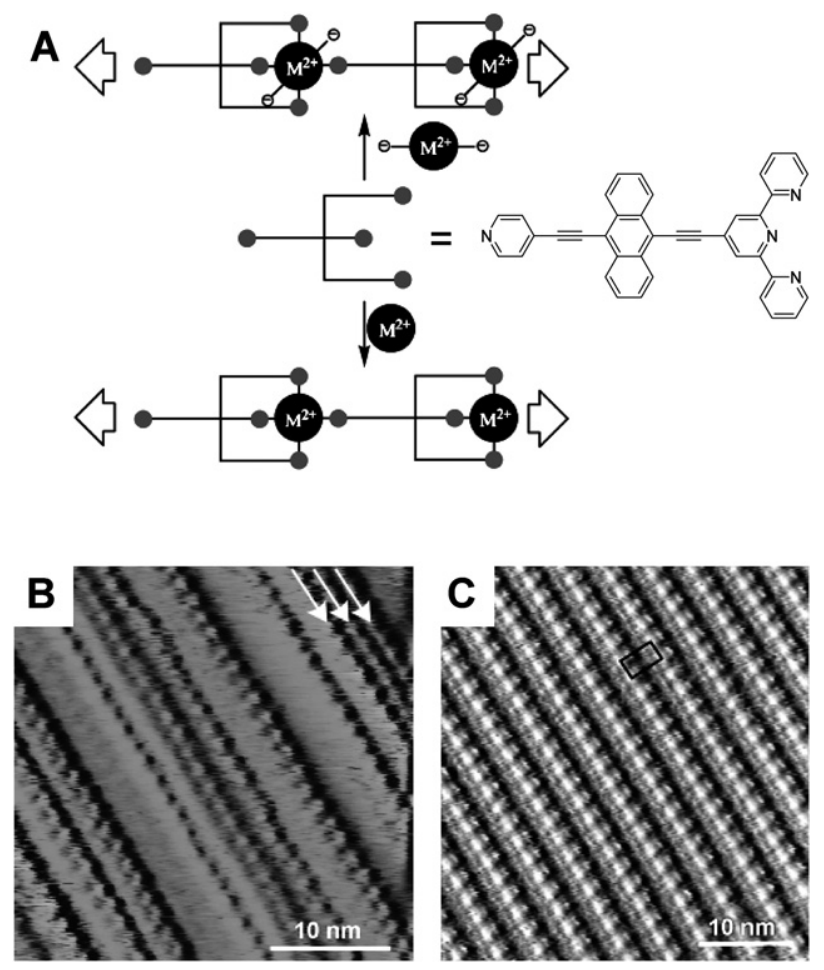

Fig. 2 (A) Schematic representation of the use of molecular tectons and metal centres to form 1D coordination polymers. STM images of the network generated by (B) the combination of the tecton and $\mathrm{CoCl}_{2}$, and (C) the combination of the tecton and $\mathrm{Pd}^{\mathrm{II}}$. (Reproduced with permission from ref. 9. Copyright Wiley-VCH Verlag GmbH and Co. KGaA). 
such as metal-ligand polymers ${ }^{9}$ or $2 \mathrm{D}$ grids, ${ }^{10-12}$ are still rather scarce. As a recent example we highlight the use of molecular tectonics to create coordination networks that form 1D and 2D arrays on graphite. ${ }^{9}$ When organic tectons containing one monodentate and one tridentate coordination pole were combined with either a neutral octahedral $\mathrm{CoCl}_{2}$ complex or a dicationic square planar $\mathrm{Pd}^{\mathrm{II}}$ species, straight $1 \mathrm{D}$ coordination polymers were formed, which in the case of the latter extend to regular 2D lattices (Fig. 2).

In addition to the strongly directional supramolecular interactions mentioned above, less predictable interactions between molecules, such as dipole-dipole and Van der Waals interactions, can also play a decisive role in the outcome of a 2D self-assembly process. In particular alkyl chain interdigitation is one of the most commonly encountered motifs within organised selfassembled monolayers of organic molecules on a surface. Interactions between alkyl chains have proven to be extremely important for stabilising monolayers in a $2 \mathrm{D}$ lattice, not only by means of their interdigitation, but also by their Van der Waals interactions with the surface that runs parallel to them. Because of a high degree of structural matching, graphite surfaces in particular interact strongly with alkyl chains (see Section 1.3), and the strength of the molecule-substrate interactions can generally be controlled by varying their length.

\subsection{Influence of the solvent}

When molecules are adsorbed at a liquid-solid interface, in addition to molecule-molecule and molecule-substrate interactions, the solvent can also play an important role. So far this aspect of 2D self-assembly has been largely neglected, and insight into the role of the solvent is still in its infancy. In the case of most STM studies a solvent is chosen for practical reasons: it should dissolve the molecules; it should have a low vapour pressure in order to prevent too rapid evaporation; it should be chemically inert; it should have a low polarity and it should have a low affinity for self-adsorption. The most commonly used solvents in STM studies and some of their physical properties are summarised in Table 1. However, several studies have demonstrated that the solvent is not always an innocent factor when molecular assemblies are formed at a liquid-solid interface. The most dramatic effect a solvent can have is that it co-adsorbs with solute molecules into a multicomponent monolayer. In most of the reported cases, such a co-adsorption was a matter of serendipity, but in hindsight the participation of solvent molecules in the assembly could be easily rationalised..$^{13,14}$

In other cases, the influence of the solvent is less straightforward. A recently reported example concerns the formation of porous structures based on the self-assembly of trimesic acid

Table 1 Frequently used solvents in STM studies at the liquid-solid interface, their melting (m.p.) and boiling (b.p.) points and dielectric constants $\left(\varepsilon_{\mathrm{r}}\right.$ at $\left.293 \mathrm{~K}\right)$

\begin{tabular}{llll}
\hline Solvent & m.p. $\left({ }^{\circ} \mathrm{C}\right)$ & b.p. $\left({ }^{\circ} \mathrm{C}\right)$ & $\varepsilon_{\mathrm{r}}$ \\
\hline 1-Phenyloctane & -36 & $261-263$ & 2.26 \\
1,2,4-Trichlorobenzene & 16 & 214 & 3.95 \\
$n$-Tetradecane & 5.8 & $252-254$ & 2.06 \\
\hline
\end{tabular}
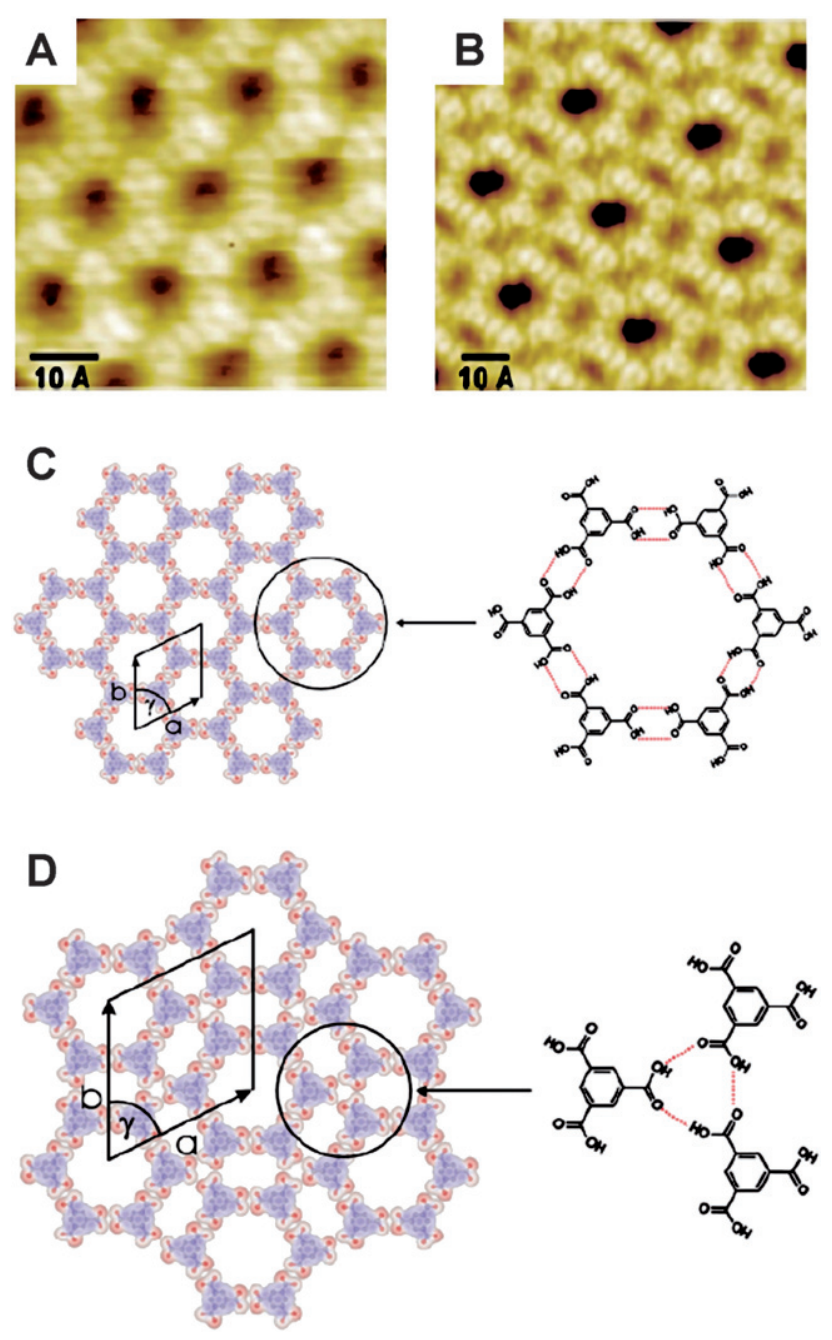

Fig. 3 STM images (in UHV) of (A) a honeycomb network and (B) a flower-like network formed by TMA on graphite. (C) Molecular model of the honeycomb structure. (D) Molecular model of the flower-like structure. (Reproduced with permission from ref. 31. Copyright WileyVCH Verlag GmbH and Co. KGaA).

(TMA) molecules at the interface of graphite and a variety of solvents. ${ }^{15}$ As a result of the planar structure of the molecule and the positioning of the trigonal exodentate functional groups, a so-called 'honeycomb' network with pores of $\sim 1.1 \mathrm{~nm}$ is frequently formed on the surface (Fig. 3A). Each of the pores is surrounded by six TMA molecules, which are all connected by double hydrogen bonding interactions between the carboxylic acids (Fig. 3C). However, this is only one of the possible polymorphs that are observed. Another, more tightly packed polymorph reveals a flower-like motif where, in addition to carboxylic acid dimers, carboxylic acid trimers are also observed (Fig. 3B and D).

By using a homologous series of alkanoic acids as solvents, ranging from butyric to nonanoic, the self-assembly of the molecules into either the flower-like or the honeycomb polymorph could be controlled. Two possible mechanisms for the observed solvent-induced polymorphism were proposed: (i) stabilisation of a specific precursor seeds by a particular solvent 
already in solution, and (ii) solvent co-adsorption and/or stabilisation of the polymorphs on the surface itself. The first proposed mechanism concerns the hydrogen bonding interactions between the carboxylic acid functionalities within the two polymorphs. In the case of the honeycomb structure, hydrogenbonded carboxylic acid dimers are the exclusively occurring motifs, while in the more closely packed flower-like structure trimers are also present. The solvents with shorter alkyl chains were proposed to favour the formation of trimer precursors, whereas the solvents with longer alkyl chains would favour dimers. Although there was no direct evidence found for the presence of these precursors, it was suggested that viscosity is one of the factors that influences the equilibrium between TMA dimers and trimers. Regarding the second proposed mechanism, it was rationalised that the small voids present in the flower-like structure would be filled and stabilised by the smaller solvent molecules. Similar solvent effects were observed for surface networks formed by the related molecule benzene-1,3,5-tribenzoic acid. ${ }^{16}$

It is clear that although some solvent-dependent aspects have been revealed, more systematic studies, supported by molecular modelling, would be invaluable in order to gain more insight into the powerful, but currently unclear role, which a solvent can play in directing or stabilising monolayers at liquid-solid interfaces.

\subsection{Influence of the substrate}

Over the years, the favoured substrate of choice to study physisorbed monolayers with STM has been highly oriented pyrolytic graphite (HOPG), because it is inert, easy to clean, and very stable under ambient conditions. However, recently the use of other flat, conductive surfaces is also gaining interest, and substrates like $\mathrm{MoS}_{2}, \mathrm{MoSe}_{2}{ }^{17}$ and $\mathrm{Au}(111)$ have proven to be very suitable for STM studies at liquid-solid interfaces. More than a decade ago these surfaces were already being used to investigate the substrate-dependent pattern formation of liquidcrystalline materials, but more recently they have been used to study in detail the ordering of physisorbed monolayers of linear alkanes and alcohols. On $\mathrm{MoS}_{2}$ the substrate-alkane interaction is very weak and only tilted lamellae of alkanes are detected, ${ }^{18}$ but on $\mathrm{Au}(111)$ alkanes and alcohols form highly ordered rows. ${ }^{19}$ Whereas on graphite alkane molecules self-assemble into similar arrays, there exists a remarkable difference between the two surfaces with respect to substrate-molecule interactions. On graphite, the rows of alkanes with odd or even numbers of carbon atoms always form an angle with the molecular axis of the surface of $90^{\circ}$, while on $\mathrm{Au}(111)$ there is a clear odd-even effect: for odd alkanes the angle is $90^{\circ}$, while for even alkanes it is $60^{\circ}$ (chain lengths <17). ${ }^{20,21}$ This difference has been attributed to a difference in commensurability of the alkane lattice with the two substrates. The length of the $\mathrm{C}-\mathrm{C}-\mathrm{C}$ zigzag $(2.54 \AA)$ is close to the atomic spacing of HOPG (the distance between two adjacent hexagons in the graphite lattice is $2.46 \AA$ ), while the nearest neighbouring atoms at the $\mathrm{Au}(111)$ surface are $2.88 \AA$ apart. Clearly, the substrate plays an often crucial role in directing the self-assembly, even for weakly adsorbed molecules. This is a curse and blessing at the same time. Compared to supramolecular chemistry in solution, it adds to the complexity of the self-assembly process. However, one can take advantage of the differences between substrates in fine-tuning the substrate templating effect for $2 \mathrm{D}$ assembly.

\section{Host-guest chemistry at liquid-solid interfaces}

Mastering the complex interplay between molecule-molecule, molecule-substrate, molecule-solvent and solvent-substrate interactions opens an enormous opportunity to form structural patterns of high complexity and functionality. A recent exciting development is the focus on multicomponent systems, especially those that involve host-guest interactions. For instance, 2D nanoporous molecular layers are designed to direct the adsorption of guest species. In many cases, the 'voids' turn out to be ideal host sites (space confinement), although sometimes guest molecules adsorb on the host skeleton rather than in the voids (specific host-guest interactions). There are basically two different approaches for constructing such 2D nanoporous layers. The first approach is based on the self-assembly of intrinsically porous molecules, the so-called macrocycles (covalently built hosts). In the second approach, intrinsically nonporous molecules self-assemble into porous $2 \mathrm{D}$ patterns. Both approaches have advantages and disadvantages, which will be discussed below.

\subsection{Covalently built hosts}

Amongst the simplest host molecules are crown ethers, which have proven themselves as selective and strong binders of positively charged ions. Kunitake et $a .^{22}$ were the first to report on the host-guest binding properties of simple dibenzo-18-crown-6 molecules in an electrochemical STM (EC-STM), a setup in which two additional electrodes are employed to gain independent control over the electrochemical potentials of tip and sample. At the interface of a $\mathrm{Au}(111)$ surface and a $0.05 \mathrm{M}$ $\mathrm{H}_{2} \mathrm{SO}_{4}$ subphase, these molecules and their inclusion complexes with potassium ions were imaged with submolecular resolution. Interestingly, the STM images of the complex could be reversibly changed at different tunnelling conditions: upon decreasing the tunnelling current from 1.0 to $0.67 \mathrm{nA}$, the free crown ether hosts remained, but the bound potassium ions were no longer observed. Also in an EC-STM, tetra-crown ether-functionalised phthalocyanines were immobilised at the interface of $\mathrm{Au}(111)$ and $0.05 \mathrm{mM}$ aqueous $\mathrm{HClO}_{4}$ (Fig. 4). ${ }^{23}$ Extended domains of this molecule could be imaged, and high resolution STM images revealed that the square features corresponding to the large aromatic cores of the molecules were each surrounded by four bright spots, which correspond to the crown ether rings. After adding an aqueous solution containing $10 \mathrm{mM} \mathrm{Ca}^{2+}$ ions dropwise to the subphase, only two of these bright spots remained visible along the diagonal of each molecule (Fig. 4C). It was proposed that the other two spots, which had turned dark in the STM image, corresponded to two crown ether rings in which $\mathrm{Ca}^{2+}$ ions were complexed. The fact that only two and not four $\mathrm{Ca}^{2+}$ ions were complexed by each phthalocyanine host is probably due to electrostatic repulsion: upon filling the remaining crown ether moieties, the bound $\mathrm{Ca}^{2+}$ ions would get too close to the crown ethers of neighbouring hosts that already contained ionic guests (Fig. 4D). A remarkable observation was that when instead of a $\mathrm{Au}(111)$ surface a $\mathrm{Au}(100)$ surface was used in the 
A

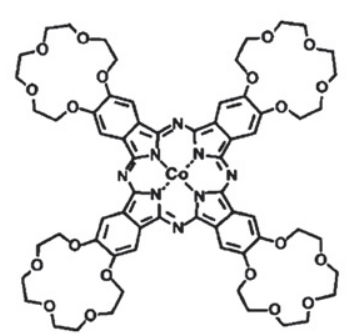

B

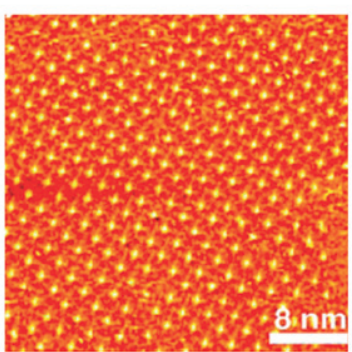

C

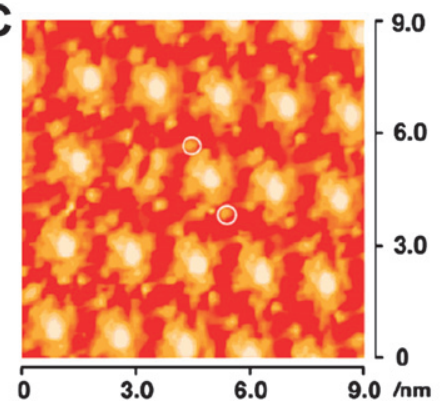

A

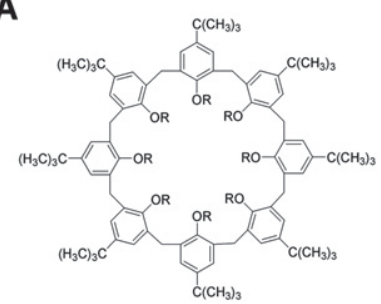

C
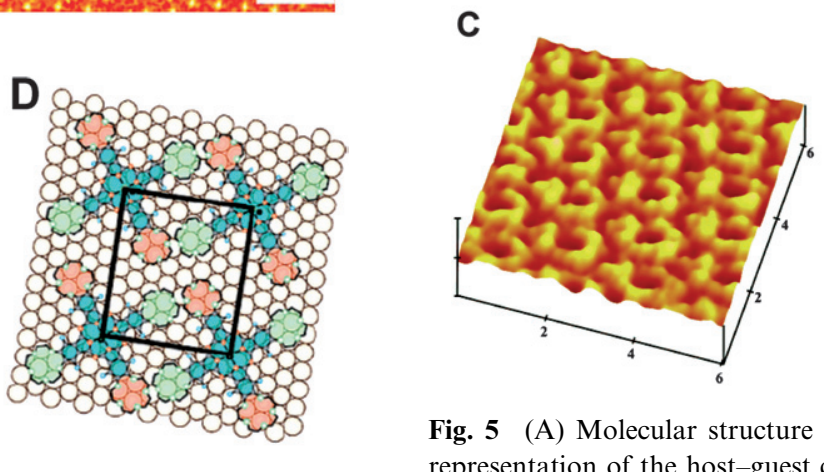

Fig. 4 (A) Molecular structure of a cobalt phthalocyanine containing four crown ethers. (B) STM image of a monolayer of this molecule at the interface of $\mathrm{Au}(111)$ and a solution of $0.05 \mathrm{mM}$ aqueous $\mathrm{HClO}_{4}$. (C) High resolution STM image of the same monolayer in the presence of $\mathrm{Ca}^{2+}$ ions. Two of the crown ethers of each phthalocyanine host a $\mathrm{Ca}^{2+}$ ion. Bright spots corresponding to unoccupied crown ether ligands are indicated by white circles. (D) Proposed structural model of the host-guest complex; the green-coloured crown ethers bind the $\mathrm{Ca}^{2+}$ ions; note that filling the two other crown ethers would probably lead to electrostatic repulsion between the positively charged ions. (Reproduced with permission from ref. 23. Copyright 2004 American Chemical Society).

host-guest experiments, no complexation of $\mathrm{Ca}^{2+}$ ions was observed. This lack of capability to bind guests was attributed to a difference in crystallographic orientation of the Au-lattice underneath the monolayers of the phthalocyanine hosts.

Larger and more complex cavity molecules have also been used as hosts in self-assembled monolayers. The bowl-shaped calix[8]arene host shown in Fig. 5A has a cavity which is large enough to accommodate a $\mathrm{C}_{60}$ fullerene guest molecule. ${ }^{24} \mathrm{In}$ EC-STM images of monolayers of the calix[8]arenes at the interface of $\mathrm{Au}(111)$ and aqueous $0.1 \mathrm{M} \mathrm{HClO}_{4}$, dark depressions are observed which can be associated with the cavities of the hosts (Fig. 5C). Monolayers of the hosts with fullerene molecules bound in their cavities at the same interface showed similar patterns in the STM image, but bound $\mathrm{C}_{60}$ guest molecules are now clearly identifiable as bright spots in the centres of the cavities (Fig. 5D).

Both the cavities of covalent hosts and their rims can be used as scaffolds for guests, thus opening the way to the construction of 3D assemblies. Cyclo[12]thiophenes, fully conjugated thiophene oligomers, were found to self-assemble at a graphite surface into porous monolayers (Fig. 6). ${ }^{25}$ The molecules adopt a spider-like orientation that lifts their aromatic ring about $0.5 \mathrm{~nm}$ above that surface (Fig. 6B). When a solution of $\mathrm{C}_{60}$ was added to such a monolayer, the fullerene molecules appeared to be occasionally captured within the
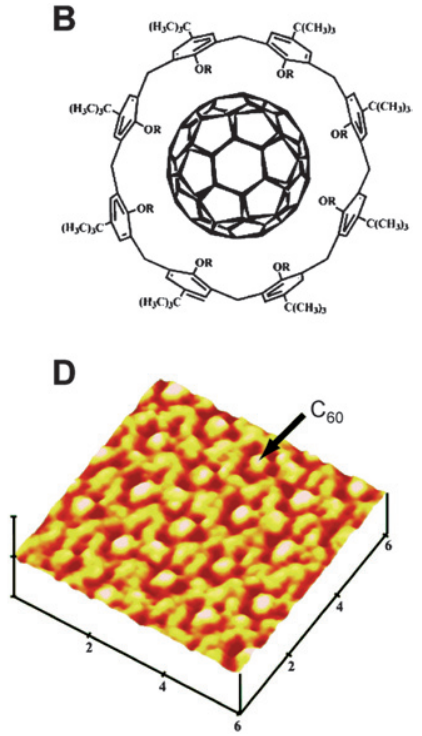

Fig. 5 (A) Molecular structure of a calix[8]arene host. (B) Schematic representation of the host-guest complex between the calix[8]arene and a fullerene. (C) STM image of a monolayer of the hosts at the interface of $\mathrm{Au}(111)$ and $0.1 \mathrm{M} \mathrm{HClO}_{4}$. (D) STM image of a monolayer of the hostguest complex with $\mathrm{C}_{60}$; The arrow points at a bound fullerene molecule. (Reproduced with permission from ref. 24. Copyright Wiley-VCH Verlag $\mathrm{GmbH}$ and Co. KGaA).
A

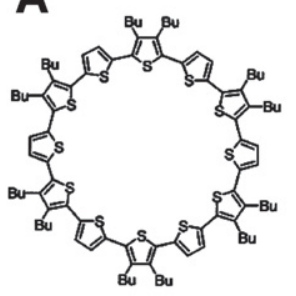

B

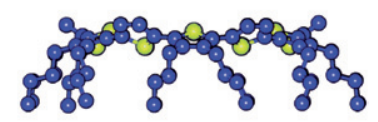

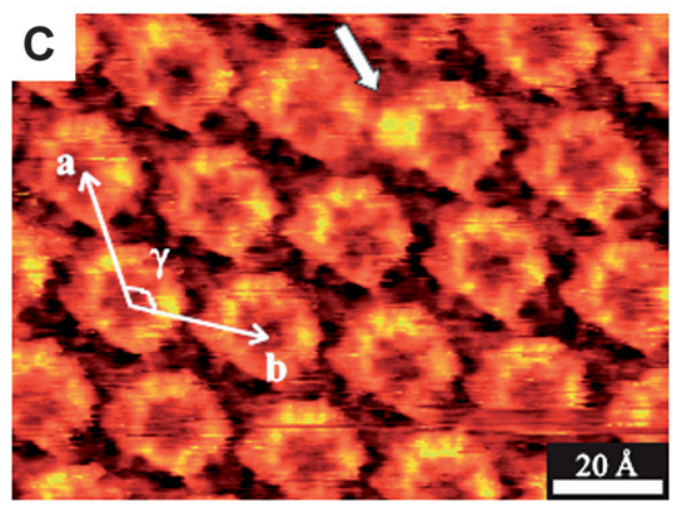

Fig. 6 (A) Molecular structure of cyclo[12]thiophene. (B) Spider-like conformation of the molecule when it is adsorbed at a graphite surface. (C) STM image of cyclo[12]thiophenes at the interface of graphite and 1,2,4-trichlorobenzene; the white arrow points to a $\mathrm{C}_{60}$ molecule bound on top of the rim of one of the macrocycles. (Reproduced with permission from ref. 25. Copyright Wiley-VCH Verlag GmbH and Co. KGaA). 
cavities of the hosts, but they bound preferentially on top of the rim of the cyclo[12]thiophenes. ${ }^{26}$ At those rims, their binding is better stabilised as a result of attractive donoracceptor interactions between the electron-rich thiophenes and the electron-poor fullerenes. Interestingly, only $1: 1$ complexes between the hosts and their guests were formed, although the aromatic ring of each cyclo[12]thiophene in principle offers multiple binding sites. The reason for the selective $1: 1$ complexation appeared to be a significant shifting of the HOMO of the underlying host towards the binding site when a fullerene was bound, thereby creating a dipole in the molecule which disfavours the complexation of additional guests. The dipole also influenced the binding properties of adjacent hosts, since due to their close proximity it created quadrupoles in these hosts which directed the binding of fullerenes at exactly the same locations.

Site-selective guest inclusion was demonstrated in networks of butadyine-bridged planar macrocycles at the graphite-liquid interface. ${ }^{27}$ Whereas the pyridine- and benzene-based macrocycles (Fig. 7A) both formed porous networks at such an interface, only the former appeared to be capable of binding charged tropylium guests, visible as bright spots in the centres of the macrocycles, as a result of ion-dipole interactions (Fig. 7B). This selectivity was further confirmed when mixed monolayers of both macrocycles were adsorbed. In the STM images, squares filled with bright spots, corresponding to pyridinophane-tropylium complexes, and darker square features, corresponding to the benzene-based macrocycles, appeared randomly within the monolayer domains.
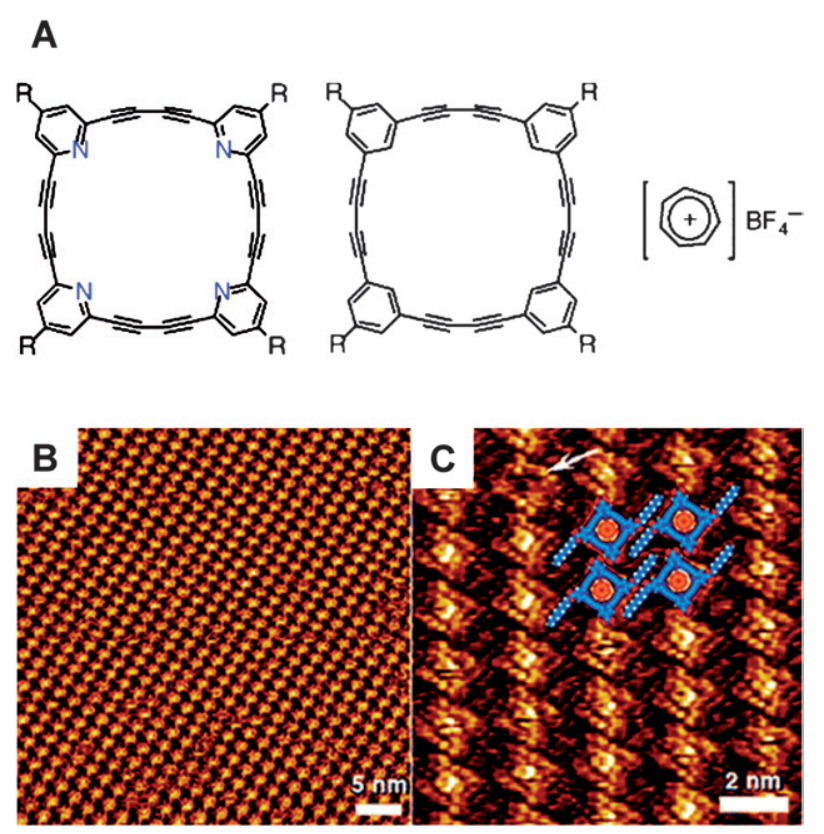

Fig. 7 (A) Molecular structures of the planar macrocycles (left and middle) and the tropylium guest (right). (B) STM image of host-guest complexes between the pyridinophane macrocycles and the tropylium guests (bright spots) at the interface of graphite and 1,2,4-trichlorobenzene- $\mathrm{CH}_{3} \mathrm{CN}-\mathrm{CHCl}_{3}(10: 9: 1)$ solution. (C) Magnified STM image with four host-guest complexes drawn in. (Reproduced with permission from ref. 27. Copyright 2008 American Chemical Society).

\subsection{Self-assembled hosts}

An alternative to the use of covalently built host molecules is to generate hosts in situ by means of supramolecular self-assembly of small components. The self-assembly of discrete porous architectures in three dimensions is often far from trivial due to unpredictable entropic factors that can play a role when several components have to be brought together into one discrete and well-defined structure. However, at a surface or interface the self-assembling components can be confined to two dimensions, and this hampers or eliminates several degrees of translational and rotational freedom of the molecules. During recent years a variety of porous self-assembled monolayers have been created and imaged with STM, but in most cases under ultrahigh vacuum conditions. ${ }^{28-30}$ A pioneering example of such a network, which is formed both under UHV and at liquid-solid interfaces, is based on the trimesic acid TMA building block (see Fig. 3). ${ }^{15,31,32}$ At the interface of graphite and 1-heptadecanoic acid, such a network was used to host fullerenes. In the absence of TMA, these molecules do not form stable assemblies at the liquid-solid interface, but after their capture in one of the pores of the network, single $\mathrm{C}_{60}$ molecules could be imaged (Fig. 8A) and even manipulated by temporarily increasing the tunnelling current from 70 to $150 \mathrm{pA}$ whilst scanning. As a result the guest was displaced from one pore into the adjacent one (Fig. 8B-D). Only displacements over one cavity per manipulation were observed, and the process was popularised as molecular "nanosoccer". ${ }^{33}$ Similarly, the flat aromatic molecule coronene
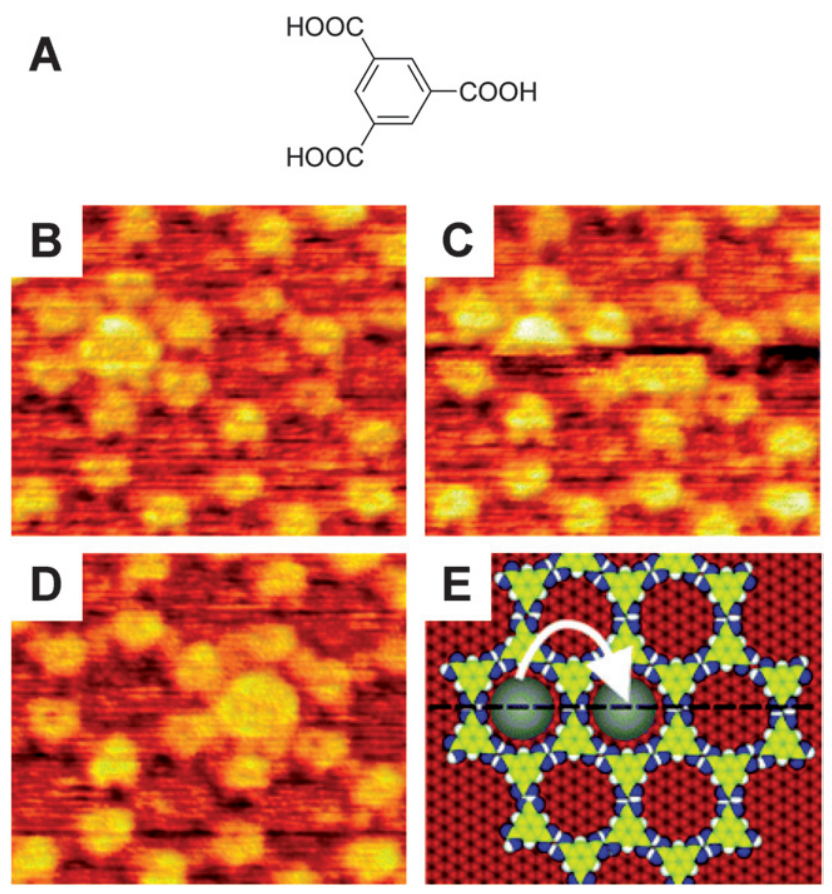

Fig. 8 (A) Molecular structure of TMA. (B) STM image of the TMA honeycomb network at the interface of graphite and 1-heptadecanoic acid in which one of the pores is occupied by a $\mathrm{C}_{60}$ molecule. (C-D) STM images of the displacement of the bound $\mathrm{C}_{60}$ molecule to an adjacent pore by manipulation with the STM tip. (E) Cartoon illustrating the transfer process. (Reproduced with permission from ref. 33. Copyright 2004 American Chemical Society). 

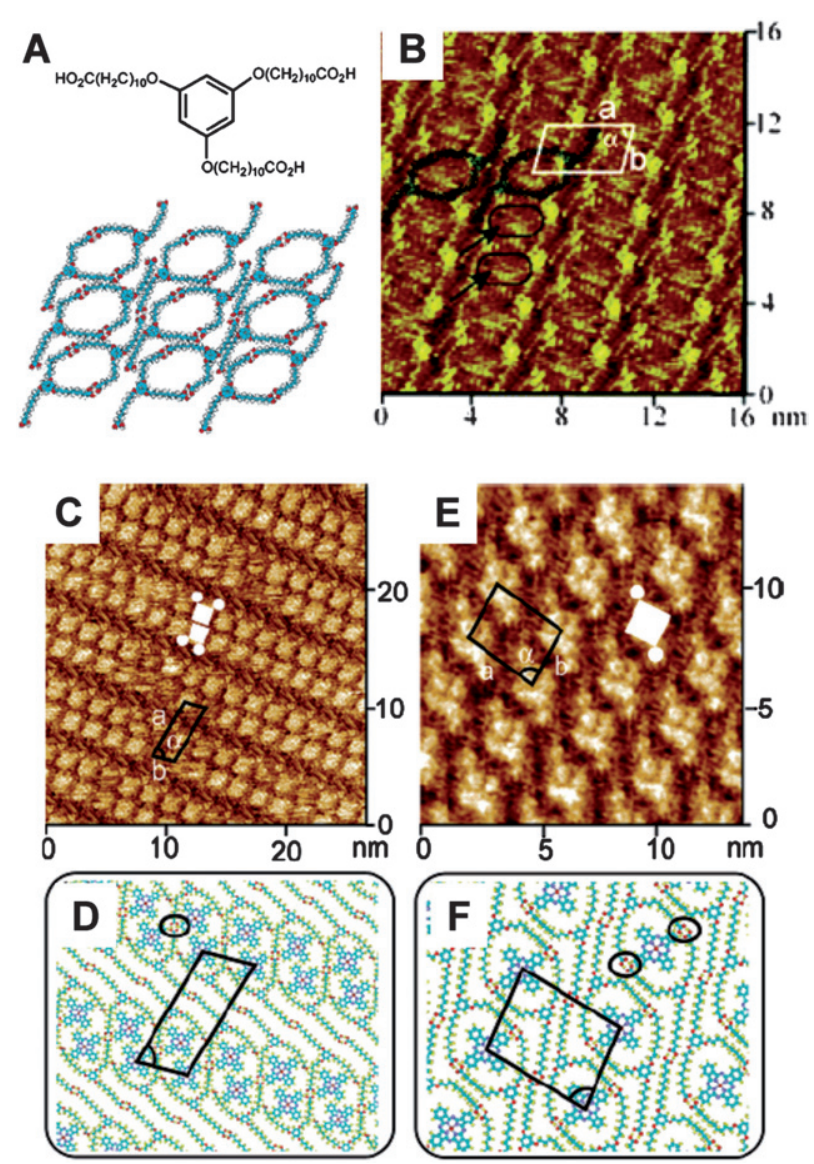

Fig. 9 (A) Molecular structure of TCDB and a model of the porous network it forms at a graphite surface. (B) STM image of the network. (C) STM image of a monolayer of the host guest complex between TCDB and VOPc, in which each pore contains two guest molecules. (D) Model of this monolayer. (E) STM image of the same monolayer after annealing at $90^{\circ} \mathrm{C}$, each of the pores now contains only one guest. (F) Model of this monolayer. (Reproduced with permission from ref. 35 and 36. Copyright 2004 and 2007 American Chemical Society).

$\left(\mathrm{C}_{24} \mathrm{H}_{12}\right.$, see Fig. 11B $)$ has been captured in the porous network formed by TMA, and this guest could also be manipulated by the STM tip, in the sense that it could be removed from the cavities. ${ }^{34}$

A derivative of TMA, 1,3,5-tris(10-carboxydecyloxy)benzene (TCDB) has three long aliphatic spacers between the core and the hydrogen-bonding functionalities. It also forms porous networks at graphite surfaces, in which two of the carboxylic acid groups of each molecule are hydrogen bonded to a neighbour to form a pseudo-rectangular pore, whereas the third carboxylic acid dimerises via hydrogen bonds with the carboxylic acid of a more distant TCDB molecule (Fig. 9A-B). ${ }^{35}$ The pores of the network, which have a dimension of $2.3 \times 1.3 \mathrm{~nm}$, were found to be good hosts for copper phthalocyanine $(\mathrm{CuPc})$ and coronene. Whereas each of the pores only captured one $\mathrm{CuPc}$ guest, a mixed monolayer of TCDB and coronene showed different domains of networks in which each pore included either one or two coronene guests. The supramolecular nature of the network of hosts should in principle allow reorganisation of the molecules at the surface by breaking and reforming of the intermolecular hydrogen bonds between the carboxylic acids. Such a reorganisation was observed when instead of $\mathrm{CuPc}$, the nonplanar guest vanadyl phthalocyanine (VOPc) was used. Upon adsorbing a mixture containing TCDB and VOPc $(2: 1)$ to HOPG at room temperature, a network was formed which predominantly filled each of its pores with two VOPc molecules (Fig. 9C-D) ${ }^{36}$ Subsequent thermal annealing of the surface, by warming it gradually from room temperature to $90{ }^{\circ} \mathrm{C}$, gave rise to an expulsion of the VOPc dimers from the cavities. Concomitantly, the molecules of the host network reorganised into a differently arranged structure, containing pores to which only one VOPc guest returned. Upon annealing at $90{ }^{\circ} \mathrm{C}$ for a longer time, the thermodynamically favoured network containing one VOPc per pore was obtained exclusively (Fig. 9E-F). Close inspection of the STM images of the $1: 1$ complex, in combination with DFT calculations, revealed that in this case the two TCDB molecules forming a pore are connected by two dimers of hydrogen bonded carboxylic acid functionalities, whereas in the pores that contain two VOPc guests at lower temperature, only one of these dimeric interactions is retained. Extensive DFT calculations and STM studies of a series of $1: 1$ and $1: 2$ host-guest complexes between the TCDB network and phthalocyanine guests confirmed that a delicate balance between a large number of intermolecular interactions determines the overall stability and 2-dimensional structure of the resulting multicomponent monolayer. ${ }^{37}$

Recently, the TCDB network has been applied as a template to organise "supramolecular rectangles." ${ }^{38}$ Whereas these molecules were found to form only disordered layers at graphite surfaces, ${ }^{39,40}$ in the presence of a TCDB network they were distributed over the surface as monodisperse entities as a result of their perfect fit within the pores. Related "supramolecular squares" with dimensions larger than the pores were not bound within the network, which highlights the substrate selectivity of the nanoporous surface.

2D honeycomb-like networks can also be formed exclusively by Van der Waals interactions between interdigitating alkyl chains. Alkyl-substituted annulenes have been shown to be excellent building blocks for building a variety of $2 \mathrm{D}$ networks at a liquid-solid interface. ${ }^{41,42}$ In addition, these networks were suitable for the binding of guest molecules. At the interface of graphite and 1,2,4-trichlorobenzene, annulene derivatives 1a and 1b formed extended monolayers containing pores with an ideal size to host flat aromatic molecules, such as coronene (Fig. 10AB). At relatively high concentrations, ${ }^{43}$ annulenes with longer alkyl chains, such as 1c and 1d, did not form porous networks, but instead they self-assembled into much tighter packed linear arrays. In the case of compound 1c, porosity could be induced by the addition of guest molecules, which reorganise the $2 \mathrm{D}$ monolayer structure and are selectively included therein. ${ }^{44}$ This behaviour, which is reminiscent of induced-fit binding in natural and artificial hosts, has been demonstrated by the addition of a solution of coronene in 1,2,4-trichlorobenzene to a selfassembled monolayer of 1c on graphite. Dramatic changes in monolayer structure were observed and a honeycomb network was formed in which multiple coronene molecules could be included as guests. Apparently, the presence of these guests renders the honeycomb-like structure thermodynamically more favourable than the linear arrays. Molecular modelling and host-guest titrations (STM images 1-4 in Fig. 10C) at the 

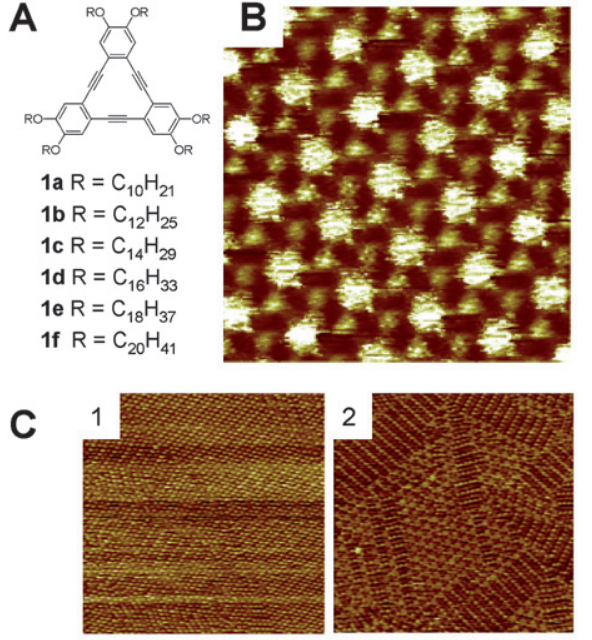

$G / H=0: 1$

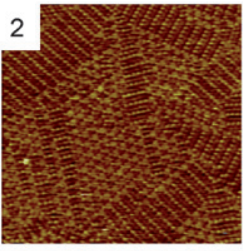

$\mathrm{G} / \mathrm{H}=2: 1$

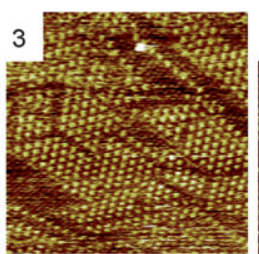

$\mathrm{G} / \mathrm{H}=\mathbf{4}: 1$

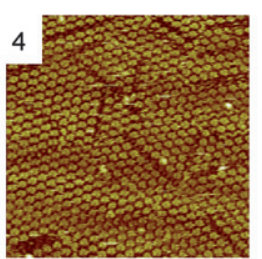

D

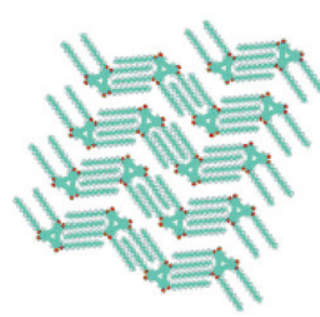

Linear Structure

E

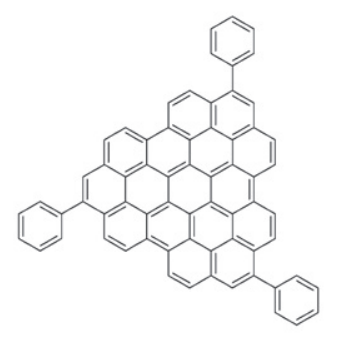

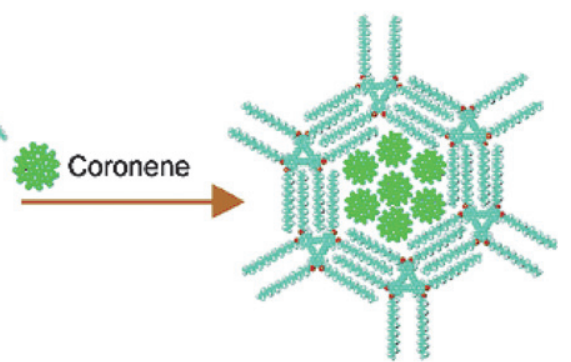

Honeycomb Structure

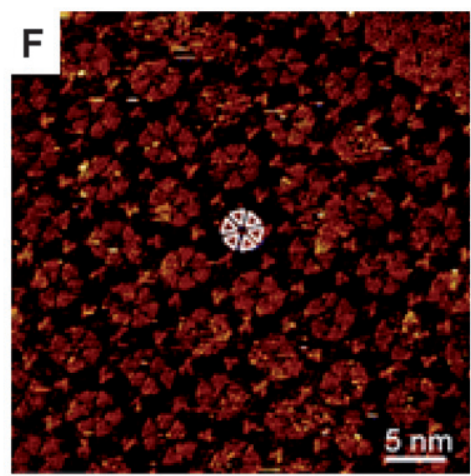

Fig. 10 (A) Molecular structure of annulene derivatives 1a-1d. (B) STM image $\left(16 \times 16 \mathrm{~nm}^{2}\right)$ of a porous network formed by 1a at the interface of graphite and 1,2,4-trichlorobenzene, with coronene guest molecules bound in the pores (bright disks). (C) STM images $\left(96 \times 96 \mathrm{~nm}^{2}\right)$ of a host-guest titration at the liquid-solid interface in which coronene is added to a self-assembled monolayer of 1c; (1) linear arrays in the absence of a guest; (2-4) gradual addition of more coronene. (D) Models explaining the induced transformation of a linear structure of the monolayer into a honeycomb-like one, in which 7 coronene guests are simultaneously bound in one pore. (E) Molecular structure of a nanographene guest. (F) STM image of a porous network of $\mathbf{1 f}$ filled with clusters of nanographene guests. (Reproduced with permission from ref. 44. Copyright Wiley-VCH Verlag GmbH and Co. KGaA, and from ref. 45. Copyright 2008 American Chemical Society).

liquid-solid interface suggested that at most seven coronene guests could be included in one cavity surrounded by six annulene molecules 1c (Fig. 10D). In addition to coronenes, other flat, planar guest molecules with large aromatic $\pi$-surfaces, such as phthalocyanines, could also induce reorganisation of the linear network formed by 1c into a honeycomb-like one. Small aromatic guests or non-planar ones did not display this effect, which highlights the remarkable substrate selectivity of the monolayer system. The structural transformation was explained by an energy gain resulting from molecule-substrate interactions between the physisorbed hosts and guests and the graphite surface, which overcomes the instability related to the presence of voids and the lower density of the host matrix when compared to densely packed linear structures. In contrast to the dynamic behaviour exhibited by $\mathbf{1 c}$, the addition of coronene to a linear network formed by annulenes 1d-1f, molecule with even longer alkyl chains, did not induce the formation of a honeycomb-like network, nor were guest molecules co-adsorbed. In this case the adsorption energy of the molecule on the graphite surface and the energy involved with intermolecular van der Waals interactions is too high to be overcome by inclusion of the guests and the conversion into a porous network is unfavourable. Such a conversion though was recently realised by using larger aromatic guest molecules. ${ }^{45}$ 'Nanographenes' (Fig. 10E), large guests with a high affinity for graphite, were found to convert densely-packed linear networks of even the annulene with the longest alkoxy chains (1f) into honeycomb-like networks, at low guest/host ratios. Giant pores with sizes up to $5.4 \mathrm{~nm}$ were observed and amounts of one up to maximal six nanographene guests could be trapped (Fig. 10F). Because of the relatively weak intermolecular interactions between the interdigitating alkyl chains, the host network was capable of changing its structure in order to accommodate the guest clusters and, as a result of this flexibility, the number of guests bound in each cavity varies.

In related work in which network formation is governed by interdigitation of alkyl chains, a tri-stilbene derivative selfassembled into a porous $2 \mathrm{D}$ network at the interface between graphite and 1-phenyloctane (Fig. 11A and C). ${ }^{46,47}$ The pores, which had diameter of approximately $1.3 \mathrm{~nm}$, were used as cavities for binding a series of flat aromatic guest molecules, which varied in shape and size (Fig. 11B). All of these guests were found to bind in the surface cavities, and appeared as bright features in the STM images (Fig. 11D-E). Remarkably, when monolayers were studied in which not all the cavities were filled, the relatively smaller guests, such as coronene, BPL and BPP, were able to diffuse from one cavity to an adjacent one, whereas the perfectly fitting guest $\mathrm{HBC}$ did not display such dynamic behaviour. Statistical investigations in combination with variable temperature experiments revealed that the diffusion of the guests is a thermally activated process that occurs within the monolayer in two dimensions, and not simply an exchange between guests 


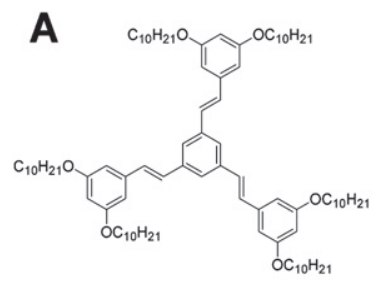

B
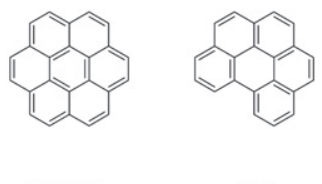

coronene

BPL

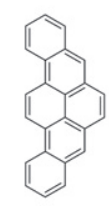

BPP

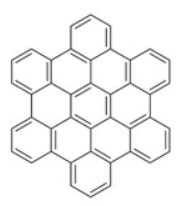

HBC
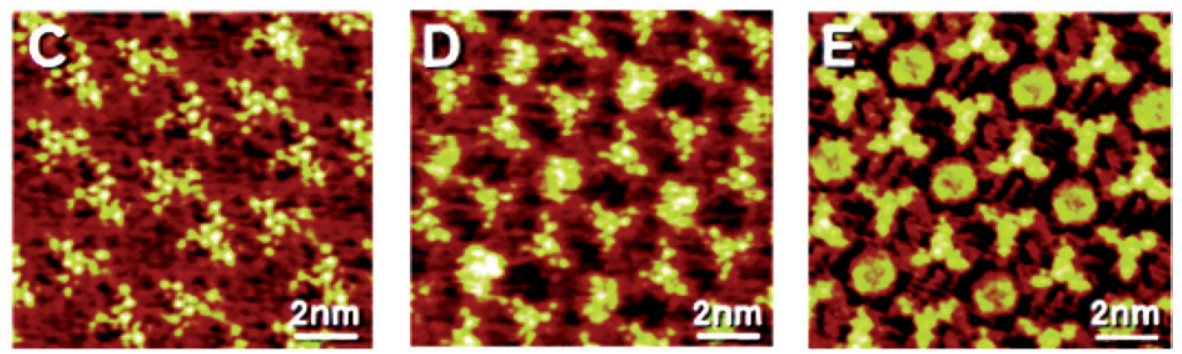

Fig. 11 (A) Molecular structure of the tri-stilbene derivative. (B) Molecular structures of the guests used for binding in the porous network. STM images of (C) the porous network formed by the tri-stilbene derivative at the interface of graphite and 1-phenyloctane, (D) the porous network in the presence of coronene, and (E) the porous network in the presence of HBC. (Reproduced with permission from ref. 47. Copyright 2006 American Chemical Society).

captured at the surface and guests dissolved in the subphase. The name '2D molecular sieve' was postulated, because only guest molecules with relatively small sizes were able to diffuse. The proposed diffusion mechanism was supported by experiments on dry layers under UHV, where similar dynamic movement of guest molecules appeared to take place.

Recently, a completely different type of network in which large aromatic guests can be captured was reported. An end-

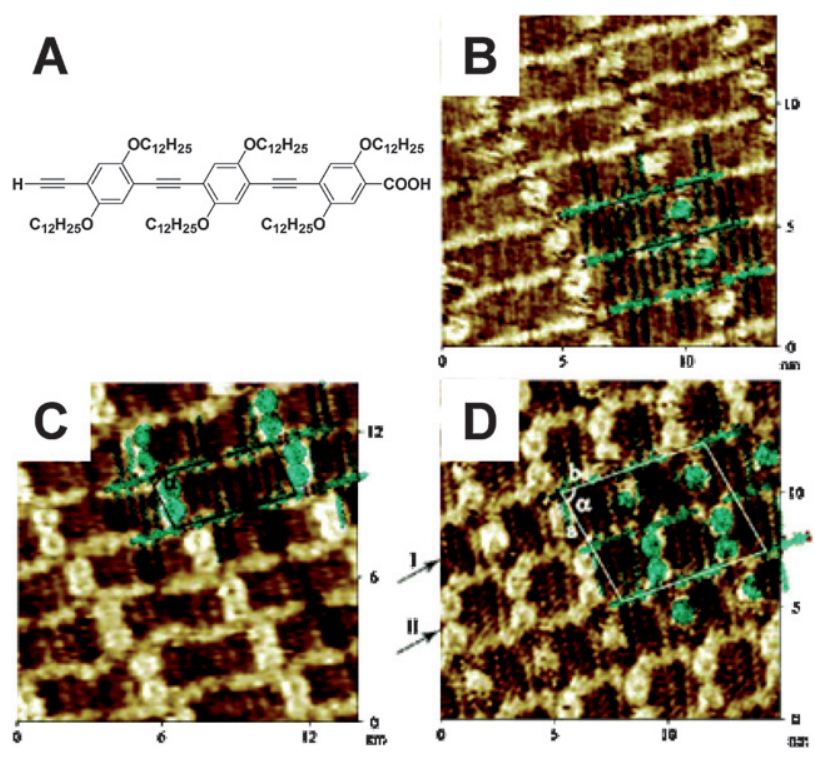

Fig. 12 (A) Molecular structure of the OPE. STM images of (B) a monolayer formed by OPE and coronene in a ratio of $2: 1$, (C) a monolayer with a ratio of $1: 1$, and (D) a monolayer with a ratio of $2: 3$. (Reproduced with permission from ref. 48. Copyright 2003 American Chemical Society). functionalised oligo(phenylene-ethynylene) (OPE, Fig. 12A) formed porous self-assembled monolayers on graphite, which were characterised by bright strands corresponding to the conjugated backbones of the molecules, separated by dark areas where the alkyl chains had adsorbed. ${ }^{48}$ The monolayer appeared to be an excellent template for the adsorption of coronene, which is bound on top of or in between the alkyl chains. By changing the ratio between the templating molecule and coronene, the distribution of the guest over the network could be precisely controlled. At a ratio OPE:coronene of $2: 1$, a 'one-by-one' distribution of the guest was observed, meaning that between two strands of the template one coronene molecule was bound (Fig. 12B). Similarly, a $1: 1$ ratio led to a $1: 2$ distribution, in which the coronene guests were organised in adjacent pairs (Fig. 12C), and a $2: 3$ ratio gave a $2: 1$ distribution (Fig. 12D). The same $2 \mathrm{D}$ porous monolayer was also capable of adsorbing tripeptides in its vacancies.

Considering the above-mentioned examples, it is clear that both approaches to constructing porous $2 \mathrm{D}$ surfaces for the binding of guest molecules, i.e. using preformed cavity-molecules or creating pores in situ by the self-assembly of smaller building blocks, can be quite successful. An important limitation of the covalent approach remains the time-consuming and generally complex synthesis of the cavity molecules. Moreover, the introduction of modifications into the molecules is often not straightforward. This is a major handicap when it comes to tuning the size of the pores. In contrast, the self-assembly approach makes use of small molecules that are in general relatively easy to synthesise and modify. On the other hand, a careful design of these building blocks is required so that they self-assemble into a desired and well-defined porous monolayer, and although supramolecular interactions are often quite predictable, other factors that can play a role during the selfassembly process, such as entropy and the influence of the 
solvent and the surface, are not always as easy to predict and control.

\section{Molecular electronics at liquid-solid interfaces}

In addition to STM being a unique tool to study the ordering of molecules on conductive surfaces down to the atomic level, it is also highly sensitive to the electronic signature of the surface and of the molecules adsorbed on them. This property is typically expressed when molecules containing both aromatic moieties and alkyl chains are imaged; because they conduct the tunnelling current better, the aromatic moieties generally appear brighter in the STM images than the alkyl chains. ${ }^{49-51}$ As STM probes the local density of states (LDOS), it can be used as a spectroscopic tool to reveal the electronic properties of individual adsorbates or their supramolecular complexes with submolecular resolution.

Since the pioneering STM studies of conjugated poly(paraphenylene-ethynylene)s (PPEs) by Rabe and coworkers in 1999, ${ }^{52}$ interest in investigating the electronic properties of (in particular) low molecular weight monodisperse oligomers has rapidly increased. For example, well-defined covalently linked donoracceptor-donor triads $\mathbf{2 a}$, in which a central perylene diimide (PDI) acceptor is flanked by two chiral oligo( $p$-phenylenevinylene) (OPV) donor moieties, were adsorbed on the interface of graphite and 1-phenyloctane and imaged with STM (Fig. 13).$^{53}$ Because the molecules have oriented their molecular axes parallel to the interface, the extremely high spatial resolution that can be achieved by STM allows probing of the individual donor and acceptor moieties. The differences in electronic properties between these moieties appeared to be reflected in the STM images (Fig. 13B): at a strongly negative surface bias potential, the OPV parts of the molecule appeared much brighter than the central PDI unit. Upon further increasing the bias voltage to positive values, the PDI part gradually appears brighter, while at a strongly positive bias voltage the OPV parts are barely visible. The observations in the STM images were explained by considering the HOMO and LUMO energy levels of both donor and acceptor moieties, taking into account the absence of a strong electronic coupling between them. At negative bias voltage, the HOMO of the OPV moiety is close to the Fermi level of graphite and electrons tunnelling from the surface to the tip couple more strongly to this HOMO than to any other energy level. Likewise, at positive bias voltage the LUMO of the PDI unit is closer to the Fermi level of the tip than to any other states and hence affects to a greater extent the tunnelling from tip to substrate. As a result, in both cases, the respective moieties appear in the STM image as the brightest parts of the molecule. Similar rectifying behaviour ${ }^{54}$ was observed for OPV-PDI hybrids that were not covalently linked, but self-assembled at a liquid-solid interface by means of hydrogen-bonded dimerisation. ${ }^{55}$

As an extension of this work, triads containing PDI moieties with different substituents at their bay positions, which strongly influence their redox properties, were investigated. ${ }^{56}$ At the interface of graphite and 1,2,4-trichlorobenzene, triads $\mathbf{2 b}$ $(\mathrm{R}=\mathrm{H})$ and $2 \mathrm{c}(\mathrm{R}=\mathrm{Cl})$ (Fig. 13A) exhibited similar biasdependent behaviour in their STM images as compound 2a. In order to quantify this bias-dependency, Scanning Tunnelling
A

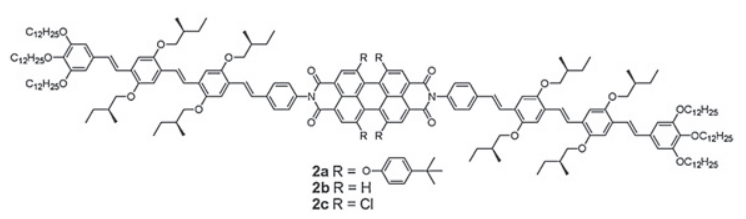

B

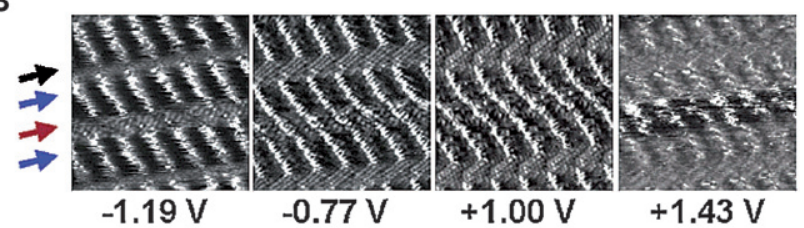

C
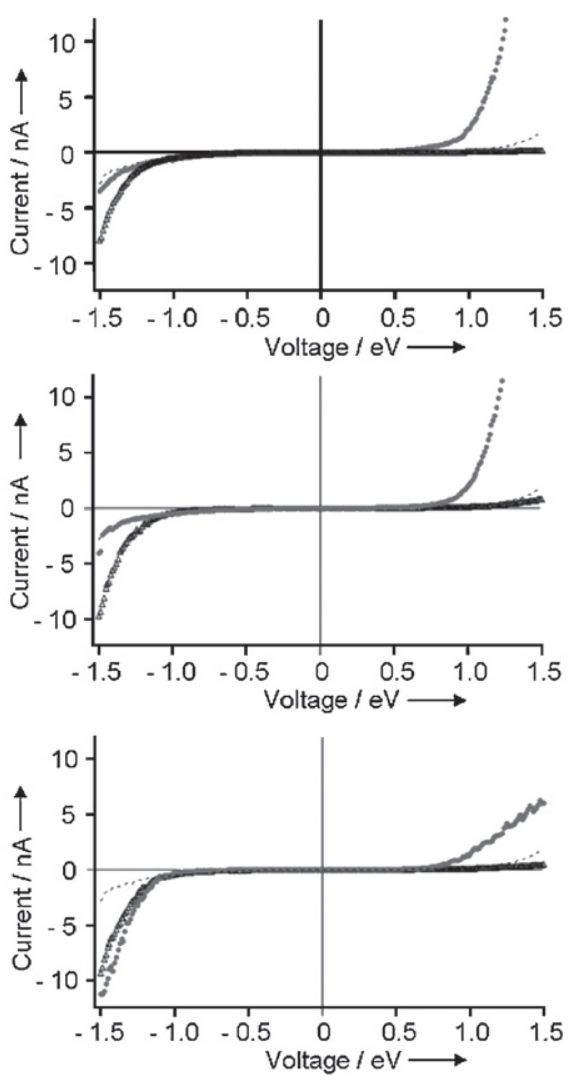

Fig. 13 (A) Molecular structure of OPV-PDI-OPV triads 2a-2c. (B) Series of STM images $\left(10.1 \times 10.1 \mathrm{~nm}^{2}\right)$ of a monolayer of $2 \mathrm{a}$ at the interface of graphite and 1-phenyloctane at varying surface bias voltage. The black arrow indicates the position of the alkyl chains, the blue arrow of the OPV moieties and the red arrow of the PDI moieties. (C) $I-V$ curves obtained on top of the alkyl chains (dashes), on top of the OPV moieties (black triangles) and PDI moieties (dark gray dots) at the graphite-1,2,4-trichlorobenzene interface; top spectrum: $I-V$ curves of pure $\mathbf{2 b}$; middle spectrum: $I-V$ curves on top of $\mathbf{2} \mathbf{b}$ in a mixed monolayer of $\mathbf{2 b}$ and $2 \mathbf{c}$; bottom spectrum: $I-V$ curves on top of $2 \mathbf{c}$ in a mixed monolayer of $\mathbf{2 b}$ and $\mathbf{2 c}$. (Reproduced with permission from ref. 56. Copyright Wiley-VCH Verlag GmbH and Co. KGaA).

Spectroscopy (STS) measurements were performed at the liquidsolid interface. In STS-mode, the STM tip is positioned on a sample coordinate of choice, the feedback loop, which controls the distance between tip and sample according to a given tunnelling current set point, is deactivated, and a current-voltage 
$(I-V)$ curve is recorded. A requirement for such a measurement is that the surface is stable. Although monolayers of $\mathbf{2} \mathbf{b}$ were stable over time, monolayers of $\mathbf{2 c}$ behaved rather dynamically, involving exchange of molecules from the surface to the liquid and vice versa. To solve this problem, mixed monolayers containing both $\mathbf{2 b}$ and $\mathbf{2} \mathbf{c}$ were constructed, in which the adsorbed molecules appeared to exchange only very slowly. In the STM images of these monolayers, the chloride-substituted triad $\mathbf{2 c}$ appeared much brighter than triad $\mathbf{2 b}$, making them easily distinguishable. The $I-V$ curves in the STS spectra of the alkyl chains were symmetric over both polarities of the bias voltage, and as expected, the STS spectra of the PDI- and OPV-parts of $\mathbf{2 b}$ in the pure and in the mixed monolayer exhibited diode-like curves, showing an inverse rectifying behaviour (Fig. 13C). Whereas the $I-V$ curve of the OPV moieties of triad 2c indicated similar diode-like behaviour as that observed for the same moieties of $\mathbf{2 b}$, the PDI part of $\mathbf{2} \mathbf{c}$ displayed a large tunnelling current for both bias polarities which is indicative of diode-like but non-rectifying behaviour. The peculiar behaviour of the chloride-substituted triad suggests that the presence of these substituents can open alternative tunnelling paths.

Another research area dealing with the development of surfaces that display molecular electronics properties is the construction of nanoscale molecular wires and circuitry, which is of particular importance with respect to miniaturisation of electronic devices. ${ }^{57,58}$ In addition to the conventional materials used to construct nanoscale wires, such as metals or carbon nanotubes, bottom-up approaches in which organic molecules self-assemble into extended one-dimensional supramolecular nanostructures are considered being versatile alternatives. ${ }^{59,60}$ TTF derivative 3 was designed to self-assemble into 1D nanostructures and at the interface of graphite and 1-octanoic acid it formed patterns of equally spaced line structures (Fig. 14). ${ }^{61}$ Based on the STM images and molecular modelling, the molecules of $\mathbf{3}$ are closely arranged as a result of intermolecular hydrogen bonding, and slightly offset $\pi-\pi$ stacking interactions

A

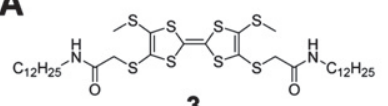

3

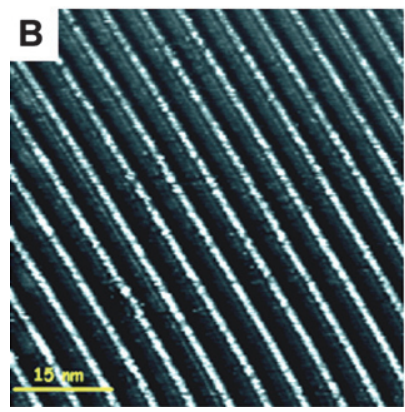

Fig. 14 (A) Molecular structure of TTF derivative 3. (B) STM image of self-assembled wires composed of $\mathbf{3}$ at the interface of graphite and 1-octanoic acid. (C) High resolution STM image. (D) Molecular model showing the arrangement of the molecules of $\mathbf{3}$ within the wires. (Reproduced with permission from ref. 61. Copyright 2006 American Chemical Society). between the aromatic TTF surfaces, which are $4.4 \AA$ apart (Fig. 14D). The presence of the amide functionalities is crucial in directing the TTF moieties to stack into columns. Quantumchemical calculations on the stacks indicated a strong interaction between the $\pi$-systems, resulting in large widths for the conductance and valence bands of the stacks, assuming that they are infinite in length. Such large bandwidths suggest that the stacks can be used for both electron- and hole-transport.

In a pioneering approach to develop a single-molecule chemical field-effect transistor (CFET), an electron-rich hexabenzocoronene (HBC) molecule covalently linked to six anthraquinone (AQ) electron acceptors was self-assembled at a liquid-solid interface. ${ }^{62}$ In the STM image of a monolayer of this compound, 4, the HBC cores are clearly visible as bright spots, and they are surrounded by less bright spots corresponding to the AQ moieties (Fig. 15). In STS experiments, the asymmetry of the $I-V$ curves taken above the HBC cores indicated clear rectifying behaviour with larger currents at negative sample bias (Fig. 15D), whereas the AQ moieties displayed rectifying behaviour at positive sample bias. When instead of neat 4, a $1: 10$ mixture of $\mathbf{4}$ and the donor 9,10-dimethoxyanthracene (DMA) was studied at the same liquid-solid interface, the STM image of the monolayer revealed a much larger unit cell in which, in addition to the spot corresponding to $\mathrm{HBC}$, six spots corresponding to donor-acceptor charge-transfer complexes between AQ and DMA were observed (Fig. 15C). The $I-V$ curve above the HBC-moieties of $\mathbf{4}$ in this case was much more symmetrical than that of the previously observed $I-V$ curve in the absence of DMA (Fig. 15D). However, upon shifting the former curve to large sample bias by $0.12 \mathrm{~V}$, followed by normalisation, a nearly perfect overlap was obtained. The observed effect is attributed to a relative shift between the Fermi level of the substrate and the molecular orbitals of the adsorbate as a result of the formation of a dipole at the interface. Because of the covalent connection of the CT complexes to the HBC core, the substrate work function is shifted by $0.12 \mathrm{~V}$. This proof-ofprinciple system can thus be viewed as a prototype CFET, based on a single molecule with an integrated nanometre-sized gate (Fig. 15E).

\section{Reactivity at liquid-solid interfaces}

At the heart of chemistry has always been the study of chemical reactions, and over the past decades numerous analytical tools have been developed to elucidate reaction mechanisms. Conventional techniques such as NMR, UV-vis spectroscopy and mass spectrometry are all very useful, yet they have the important limitation that they measure ensembles of millions of molecules at the same time and thus give only an average picture of a reaction mechanism. Such a picture can be incomplete and might be misleading, because certain molecules might react whilst others are inactive. Recently fluorescence microscopy has been applied to monitor, by single turnover counting, chemical reactions carried out on crystal facets ${ }^{63}$ and by enzymes on a surface. ${ }^{64,65}$ The importance of studying chemical reactions at a surface has very recently been recognised by Ertl being awarded the Nobel Prize in chemistry. As part of this research he has explored in detail molecular mechanisms for the catalytic synthesis of ammonia over iron and the catalytic oxidation of 
A
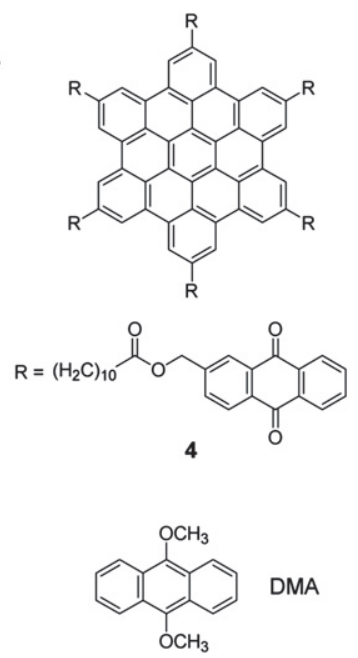

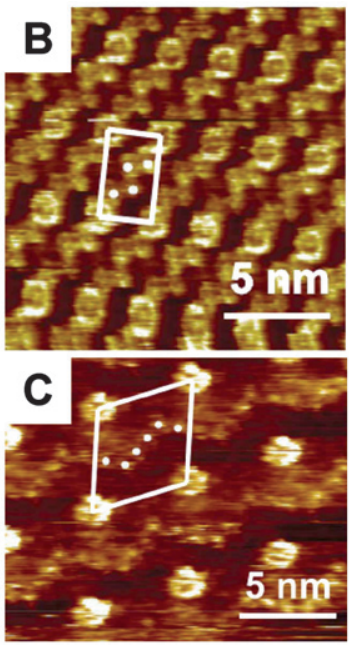

D

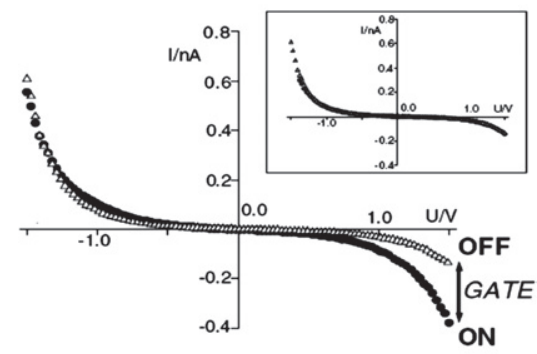

E

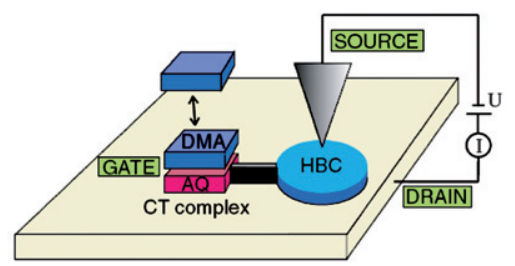

Fig. 15 (A) Molecular structures of $\mathbf{4}$ and DMA. (B) STM image of $\mathbf{4}$ at a graphite surface. (C) STM image of a $1: 10$ mixture of 4 and DMA. (D) $I-V$ curves taken above the HBC cores of neat $\mathbf{4}$ (open triangles) and above these cores where CT complexes are present between the AQ moieties of $\mathbf{4}$ and DMA (solid circles); inset: shifted and normalised data (see text). (E) Cartoon illustrating the prototype CFET. Reprinted with permission from ref. 62. Copyright (2004) by the American Physical Society.

carbon monoxide over palladium. Amongst others ${ }^{66-68}$ he was also one of the pioneers to study such dynamic processes by imaging them using STM. ${ }^{69}$ A variety of elementary chemical reactions have been investigated using STM, as well as more sophisticated ones in which the breaking and formation of chemical bonds, in some cases induced by the STM tip, is imaged in real-time and -space. ${ }^{70-72}$ Nearly all of those reactions were carried out under extreme conditions, such as UHV, high pressure, or ultra-low temperature. However, such conditions are far removed from the environments in which chemical and biological reactions usually take place, i.e., in a liquid under ambient conditions. So far, only a limited number of examples have been reported in which a chemical reaction was studied with STM under ambient conditions.

Let us start with a metal-ligand complexation reaction at the liquid-solid interface. A 4,4'-bipyridine derivative, which contains two long aliphatic chains to ascertain strong adsorption
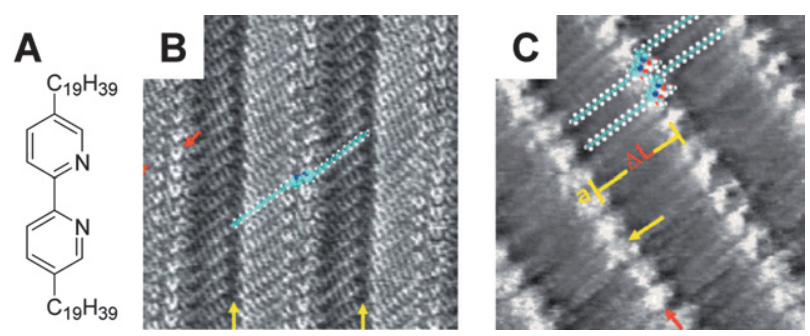

Fig. 16 (A) Molecular structure of the bipyridine derivative. (B) STM image $\left(9.1 \times 9.1 \mathrm{~nm}^{2}\right)$ of a monolayer of this molecule at the graphite-1phenyloctane interface; one modelled molecule is superimposed in the image; the red arrows point to the aromatic rings of the bipyridine moiety, while the yellow arrows indicate the lamella boundaries. (C) STM image $\left(10.2 \times 10.2 \mathrm{~nm}^{2}\right)$ of the monolayer after the addition of $\mathrm{Pd}(\mathrm{OAc})_{2}$; two modelled complexes are superimposed in the image. (Reproduced with permission from ref. 73. Copyright 2002 The Royal Society of Chemistry). to a HOPG surface, self-assembled into extended lamellar arrays at the interface between HOPG and 1-phenyloctane (Fig. 16). ${ }^{73}$ When a droplet of a solution containing $\mathrm{Pd}(\mathrm{OAc})_{2}$ in 1-phenyloctane was placed on top of this monolayer, a spontaneous change in molecular organisation was observed in situ. The angle between the alkyl chains and the lamellar axis changed, while simultaneously the lamellar distance decreased, indicating that interdigitation of the alkyl chains occurred. In addition, as a result of the accommodation of the $\mathrm{Pd}(\mathrm{OAc})_{2}$ moieties between the molecules upon their complexation to the bipyridine ligands, the distance between the molecules within the lamellae increased from 6.9 to $9.4 \AA$. In closely related work a slightly different bipyridine ligand was coupled to $\mathrm{PdCl}_{2}{ }^{74}$ In this case, two discrete reorganisation steps of the monolayer could be monitored in real-time, first a loss of interdigitation of the alkyl chains, which was followed by a structural rearrangement of the molecules within the lamellae. Note that a reaction as such does not necessarily take place on the substrate. It is more likely that bipyridine ligands in the solution phase react prior to the ones adsorbed on the substrate. Reaction product accumulates in the solution phase and due to a concentration gradient, non-reacted reagent will desorb and the reaction product will adsorb.

This is in contrast with pioneering work in the late 1990s involving the topochemical polymerisation of diacetylene compounds at the air-solid and liquid-solid interface, using the STM tip or an external stimulus such as light as the initiator of the reaction. ${ }^{75,76}$ This approach strongly relies on a careful preorganisation of the monomers at the interface, with their reactive groups positioned in close proximity in the desired orientation. In related work cinnamate derivatives physisorbed on HOPG were subjected to a light-induced [2 +2$]$ photodimerisation, resulting in a change in monolayer structure and tunnelling contrast. ${ }^{77}$

A more recent example of a polymerisation involved the controlled polymerisation of aniline at the liquid-solid interface of $\mathrm{Au}(111)$ and an aqueous $0.1 \mathrm{M} \mathrm{H}_{2} \mathrm{SO}_{4}$ solution, which was 


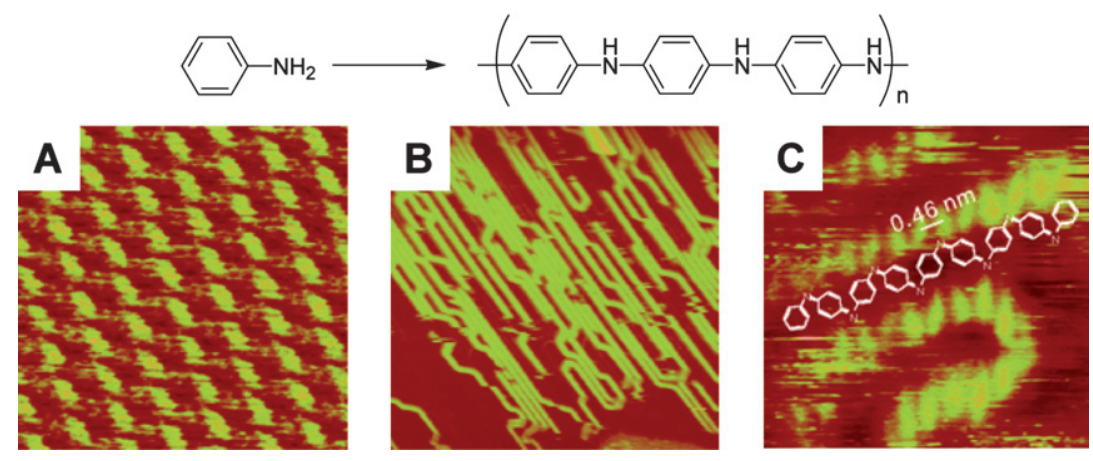

Fig. 17 Molecular structures of aniline and polyaniline and EC-STM images of the polymerisation of aniline at the interface of Au(111) and an aqueous $0.1 \mathrm{M} \mathrm{H}_{2} \mathrm{SO}_{4}$ solution. (A) Monolayer of aniline molecules at $0.9 \mathrm{~V} ; 7 \times 7 \mathrm{~nm}^{2}$. (B) The monolayer after raising the potential to $1.05 \mathrm{~V} ; 50 \times 50 \mathrm{~nm}{ }^{2}$. (C) Zoomed-in image showing the submolecular structure within the polymer chains in which the aniline monomers are arranged in a zig-zag fashion; $6 \times 6$ $\mathrm{nm}^{2}$. (Reproduced with permission from ref. 78. Copyright 2007 American Chemical Society).

carried out in an EC-STM (Fig. 17). ${ }^{78}$ Upon increasing the potential applied to a surface, on which a monolayer of aniline molecules was present, from 0.9 to $1.05 \mathrm{~V}$, an oxidative polymerisation was induced which caused a drastic change in the surface topography: elongated linear polyaniline chains with lengths up to $50 \mathrm{~nm}$ were formed. The length of the lines suggested that they consisted of approximately 100 monomers of aniline. Remarkably, the polymerisation process appeared to be strongly anisotropic, because a distinct preferential and unprecedented alignment of the polyaniline chains in the $<121>$ direction of the $\mathrm{Au}(111)$ surface was observed. Magnifications of the lines revealed their internal structure as a zig-zag ordering of head-to-tail connected monomers within the polymer chains.

Recently, for the first time a multi-step oxidation reaction, in which manganese porphyrin catalysts were adsorbed at a liquidsolid interface of $\mathrm{Au}(111)$ and $n$-tetradecane, was imaged in situ with an STM equipped with a liquid cell and a bell-jar (Fig. 18). ${ }^{79}$ Upon adding molecular oxygen $\left(\mathrm{O}_{2}\right)$ to the bell-jar it was observed that a reaction occurred with the bound catalysts, of which the apparent height increased by a factor of 3 (Fig. 18C, middle image). Statistical analysis of the STM images revealed that there was a high abundance of pairs of adjacent catalysts that had reacted with $\mathrm{O}_{2}$, which led to the suggestion that each molecule of $\mathrm{O}_{2}$ had dissociated in a homolytic fashion, thereby distributing both its oxygen atoms over catalyst neighbours. The observed reaction of the manganese porphyrins with $\mathrm{O}_{2}$ was surprising, because in $n$-tetradecane solution these catalysts were found to be completely inert. It was therefore proposed that their adsorption to the gold surface played an essential role in activating them to react with $\mathrm{O}_{2}$. For such a reaction to occur, generally a reduction of the manganese centre in the porphyrin from $\mathrm{Mn}^{\mathrm{III}}$ to $\mathrm{Mn}^{\mathrm{II}}$ is required. In situ surface reflection UV-vis
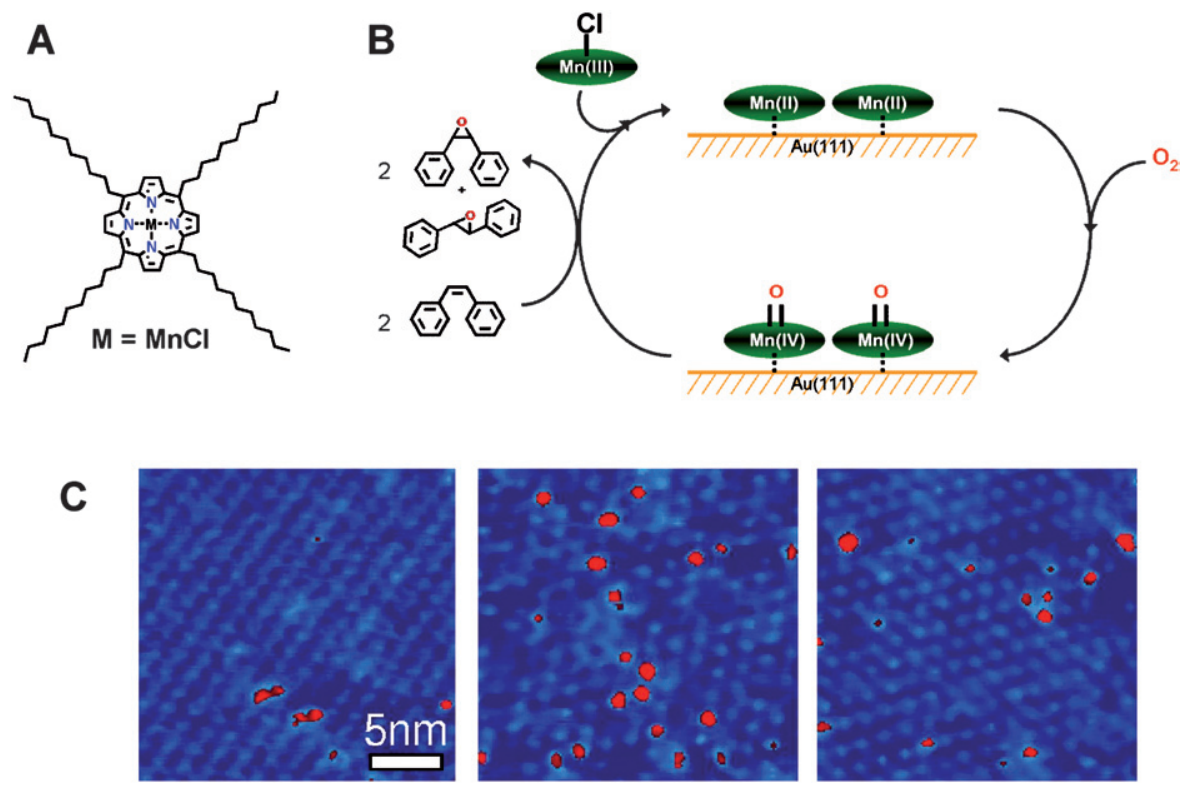

Fig. 18 (A) Molecular structure of the manganese porphyrin catalyst. (B) Proposed catalytic cycle. (C) Series of liquid cell STM images of a monolayer of the catalysts self-assembled at the interface of $\mathrm{Au}(111)$ and $n$-tetradecane; left: system under argon; middle: 4 hours after flushing the bell jar with $\mathrm{O}_{2}$, causing $\sim 10 \%$ of the catalysts to be oxidised (visible as red spots); right: 3 hours after the addition of cis-stilbene, showing a decrease in the number of oxidised catalysts. 
measurements at the liquid-solid interface suggested that, upon adsorption of the catalysts to the surface, such a reduction indeed took place, and that is was accompanied by an elimination of the chloride axial ligand (Fig. 18B). Reflectance UV-vis measurements confirmed the presence of porphyrin $\mathrm{Mn}^{\mathrm{IV}}=\mathrm{O}$ species after the reaction of the surface-bound catalysts with $\mathrm{O}_{2}$. It was concluded that their adsorption to a surface must also play an essential role in the homolytic dissociation of $\mathrm{O}_{2}$, because the occurrence of such a mechanism had previously only been observed under extreme conditions (high pressure and temperature). Because it is well known that porphyrin $\mathrm{Mn}^{\mathrm{IV}}=\mathrm{O}$ species are excellent catalysts for the epoxidation of alkenes, the catalytic surface was used in a subsequent reaction step in the epoxidation of an alkene, which was added to the liquid subphase (Fig. 18B). At the moment that this alkene, cis-stilbene, arrived at the liquidsolid interface, the number of oxidised manganese porphyrin catalysts instantaneously decreased, suggesting that they transferred their oxygen atoms to the alkene (Fig. 18C, right image). Gas-chromatographic analysis on a sample taken from the liquid cell after 4 days proved that indeed a considerable amount of cis-stilbene epoxide had been formed.

\section{Conclusions and outlook}

Since the invention of scanning tunnelling microscopy about 25 years ago, it has become almost a standard technique in many laboratories. An absolutely thrilling aspect is that it was soon realised that STM could be useful for more than just looking at atomic detail on surfaces under UHV conditions. The simplicity of the key operational principle opened the doors to exploring different systems and environments, such as the liquid-solid interface. Though a lot still needs to be explored as far as the architectural aspects of these physisorbed self-assembled layers are concerned, research efforts have shifted to designing more complex and functional surfaces. An exciting development is the real-space and -time exploration of chemical reactions at liquidsolid interfaces where substrates can even affect the outcome of the reaction path. A steadily growing number of scientists appreciate the opportunities which self-assembly at liquid-solid interfaces brings, a trend which promises this research field a bright future.

\section{Acknowledgements}

This work is supported by K.U. Leuven, the Fund of Scientific Research - Flanders (FWO), the Belgian Federal Science Policy Office through IAP-6/27, the Belgian National fund for Scientific Research (F.R.S.-FNRS), and the European Union Marie Curie Research Training Network CHEXTAN (MRTN-CT-2004512161). J.A.A.W.E. thanks the Fund for Scientific ResearchFlanders (FWO) for a postdoctoral research grant.

\section{References}

1 S. M. Clarke, Curr. Opin. Colloid Interface Sci., 2001, 6, 118.

2 G. Binnig, H. Rohrer, C. Gerber and E. Weibel, Phys. Rev. Lett., 1982, 49, 57.

3 A. Ulman, Chem. Rev., 1996, 96, 1533.

4 J. P. Rabe and S. Buchholz, Science, 1991, 253, 424.

5 S. Furukawa and S. De Feyter, Top. Curr. Chem., 2008, DOI: 10.1007/128_2007_23.

6 J. V. Barth, G. Costantini and K. Kern, Nature, 2005, 437, 671.
7 M. Lackinger, S. Griessl, T. Markert, F. Jamitzky and W. M. Heckl, J. Phys. Chem. B, 2004, 108, 13652.

8 S. Stepanow, M. Lingenfelder, A. Dmitriev, H. Spillmann, E. Delvigne, N. Lin, X. B. Deng, C. Z Cai, J. V. Barth and K. Kern, Nat. Mater., 2004, 3, 229.

9 M. Surin, P. Samorì, A. Jouaiti, N. Kyritsakas and M. W. Hosseini, Angew. Chem., Int. Ed., 2007, 46, 245.

10 M. S. Alam, S. Strömsdörfer, V. Dremov, P. Müller, J. Kortus, M. Ruben and J.-M. Lehn, Angew. Chem., Int. Ed., 2005, 44, 7896.

11 A. Semenov, J. P. Spatz, M. Möller, J.-M. Lehn, B. Sell, D. Schubert, C. H. Weidl and U. S. Schubert, Angew. Chem., Int. Ed., 1999, 38, 2547.

12 A. Mourran, U. Ziener, M. Möller, E. Breuning, M. Ohkita and J.-M. Lehn, Eur. J. Inorg. Chem., 2005, 2641.

13 W. Mamdouh, H. Uji-i, J. Ladislaw, A. Dulcey, V. Percec, F. C. De Schryver and S. De Feyter, J. Am. Chem. Soc., 2006, 128, 317.

14 P. Vanoppen, P. C. M. Grim, M. Rücker, S. De Feyter, G. Moessner, S. Valiyaveetil, K. Müllen and F. De Schryver, J. Phys. Chem., 1996, 100, 19636.

15 L. Kampschulte, M. Lackinger, A. K. Maier, R. S. K. Kishore, S. Griessl, M. Schmittel and W. M. Heckl, J. Phys. Chem. B, 2006, 110, 10829.

16 M. Lackinger, S. Griessl, W. M. Heckl, M. Hietschold and G. W. Flynn, Langmuir, 2005, 21, 4984.

17 S. Cincotti and J. P. Rabe, Appl. Phys. Lett., 1993, 62, 3531.

18 H. M. Zhang, Z. X. Xie, B. W. Mao and X. Xu, Chem. Eur. J., 2004, 10, 1415 .

19 H. M. Zhang, J. W. Yan, Z. X. Xie, B. W. Mao and X. Xu, Chem. Eur. J., 2006, 12, 4006 .

20 K. Uosaki and R. Yamada, J. Am. Chem. Soc., 1999, 121, 4090.

21 R. Yamada and K. Uosaki, J. Phys. Chem. B., 2000, 104, 6021.

22 A. Ohira, M. Sakata, C. Hirayama and M. Kunitake, Org. Biomol. Chem., 2003, 1, 251.

23 S. Yoshimoto, K. Suto, A. Tada, N. Kobayashi and K. Itaya, J. Am. Chem. Soc., 2004, 126, 8020.

24 G.-B. Pan, J.-M. Liu, H.-M. Zhang, L.-J. Wan, Q.-Y. Zheng and C.-L. Bai, Angew. Chem., Int. Ed., 2003, 42, 2747.

25 J. Krömer, I. Rios-Carreras, G. Fuhrmann, C. Musch, M. Wunderlin, T. Debaerdemaker, E. Mena-Osteritz and P. Bäuerle, Angew. Chem., Int. Ed., 2000, 39, 3481.

26 E. Mena-Osteritz and P. Bäuerle, Adv. Mater., 2006, 18, 447.

27 K. Tahara, S. Lei, W. Mamdouh, Y. Yamaguchi, T. Ichikawa, H. UjiI, M. Sonoda, K. Hirose, F. C. De Schryver, S. De Feyter and Y. Tobe, J. Am. Chem. Soc., 2008, 130, 6666.

28 J. A. Theobald, N. S. Oxtoby, M. A. Phillips, N. R. Champness and P. H. Beton, Nature, 2003, 424, 1029.

29 D. Bonifazi, A. Kiebele, M. Stöhr, F. Cheng, T. Jung, F. Diederich and H. Spillmann, Adv. Funct. Mater., 2007, 17, 1051.

30 N. Dmitriev, N. Lin, J. Weckesser, J. V. Barth and K. Kern, J. Phys. Chem. B, 2002, 106, 6907.

31 S. Griessl, M. Lackinger, M. Edelwirth, M. Hietschold and W. M. Heck1, Single Mol., 2002, 3, 25.

32 Y. Ishikawa, A. Ohira, M. Sakata, C. Hirayama and M. Kunitake, Chem. Commun., 2002, 2652.

33 S. J. H. Griessl, M. Lackinger, F. Jamitzky, T. Markert, M. Hietschold and W. M. Heckl, J. Phys. Chem. B., 2004, 108, 11556.

34 S. J. H. Griessl, M. Lackinger, F. Jamitzky, T. Markert, M. Hietschold and W. M. Heckl, Langmuir, 2004, 20, 9403.

35 J. Lu, S. B. Lei, Q. D. Zeng, S. Z. Kang, C. Wang, L. J. Wan and C. L. Bai, J. Phys. Chem. B, 2004, 108, 5161.

36 X.-H. Kong, K. Deng, Y.-L. Yang, Q.-D. Zeng and C. Wang, J. Phys. Chem. C, 2007, 111, 9268.

37 X.-H. Kong, K. Deng, Y.-L. Yang, Q.-D. Zeng and C. Wang, J. Phys. Chem. C, 2007, 111, 17382.

38 S.-S. Li, H.-J. Yan, L.-J. Wan, H.-B. Yang, B. H. Northrop and P. J. Stang, J. Am. Chem. Soc., 2007, 129, 9268.

39 J. R. Gong, L. J. Wan, Q. H. Yuan, C. L. Bai, H. Jude and P. J. Stang, Proc. Natl. Acad. Sci. U. S. A., 2005, 102, 971.

40 Q. H. Yuan, L. J. Wan, H. Jude and P. J. Stang, J. Am. Chem. Soc., $2005,127,16279$.

41 S. Furukawa, H. Uji-i, K. Tahara, T. Ichikawa, M. Sonoda, F. C. De Schryver, Y. Tobe and S. De Feyter, J. Am. Chem. Soc., 2006, 128, 3502 . 
42 K. Tahara, S. Furukawa, H. Uji-i, T. Uchino, T. Ichikawa, J. Zhang, W. Mamdouh, M. Sonoda, F. C. De Schryver, S. De Feyter and Y. Tobe, J. Am. Chem. Soc., 2006, 128, 16613.

43 S. Lei, K. Tahara, F. C. De Schryver, M. Van der Auweraer, Y. Tobe and S. De Feyter, Angew. Chem., Int. Ed., 2008, 47, 2964.

44 S. Furukawa, K. Tahara, F. C. De Schryver, M. Van der Auweraer, Y. Tobe and S. De Feyter, Angew. Chem., Int. Ed., 2007, 46, 2831.

45 S. Lei, K. Tahara, X. Feng, S. Furukawa, F. C. De Schryver, K. Müllen, Y. Tobe and S. De Feyter, J. Am. Chem. Soc., 2008, 130, 7119.

46 G. Schull, L. Douillard, C. Fiorini-Debuisschert, F. Charra, F. Mathevet, D. Kreher and A.-J. Attias, Adv. Mater., 2006, 18, 2954.

47 G. Schull, L. Douillard, C. Fiorini-Debuisschert, F. Charra, F. Mathevet, D. Kreher and A.-J. Attias, Nano Lett., 2006, 6, 1362.

48 J.-R. Gong, H.-J. Yan, Q.-H. Yuan, L.-P. Xu, Z.-S. Bo and L.-J. Wan, J. Am. Chem. Soc., 2003, 126, 12384.

49 J. Foster and J. Frommer, Nature, 1988, 333, 542.

50 D. Smith, H. Hörber, C. Gerber and G. Binning, Science, 1989, 245, 43.

51 R. Lazzaroni, A. Calderone, J.-L. Bredás and J. P. Rabe, J. Chem. Phys., 1997, 107, 99.

52 P. Samorí, V. Francke, K. Müllen and J. P. Rabe, Chem. Eur. J., 1999, 5, 2312.

53 A. Miura, Z. Chen, H. Uji-i, S. De Feyter, M. Zdanowska, P. Jonkheijm, A. P. H. J. Schenning, E. W. Meijer, F. Würthner and F. C. De Schryver, J. Am. Chem. Soc., 2003, 125, 14968.

54 A. Stabel, P. Herwig, K. Müllen and J. P. Rabe, Angew. Chem., Int. Ed., 1995, 34, 1609.

55 J. Zhang, F. J. M. Hoeben, M. J. Pouderoijen, A. P. H. J. Schenning, E. W. Meijer, F. C. De Schryver and S. De Feyter, Chem. Eur. J., 2006, 12, 9046

56 H. Uji-i, A. Miura, A. Schenning, E. W. Meijer, Z. Chen, F. Würthner, F. C. De Schryver, M. Van der Auweraer and S. De Feyter, ChemPhysChem, 2005, 6, 2389.

57 W. Schmickler and C. J. Widrig, Electroanal. Chem., 1992, 336, 213.

58 D. K. James and J. M. Tour, in Nanoscale Assembly Techniques, ed. W. T. S. Huck, Springer, New York, 2005, pp. 79-98.

59 A. P. H. J. Schenning and E. W. Meijer, Chem. Commun., 2005, 3245.

60 R. van Hameren, P. Schön, A. M. van Buul, J. Hoogboom, S. V. Lazarenko, J. W. Gerritsen, H. Engelkamp, P. C. M. Christianen, H. A. Heus, J. C. Maan, Th. Rasing, S. Speller, A. E. Rowan, J. A. A. W. Elemans and R. J. M. Nolte, Science, 2006, 314, 1433.
61 J. Puigmartí-Luis, A. Minoia, H. Uji-i, C. Rovira, J. Cornil, S. De Feyter, R. Lazzaroni and D. B. Amabilino, J. Am. Chem. Soc., 2006, 128, 12602.

62 F. Jäckel, M. D. Watson, K. Müllen and J. P. Rabe, Phys. Rev. Lett., 2004, 92, 188303

63 M. B. J. Roeffaers, B. F. Sels, H. Uji-i, F. C. De Schryver, P. A. Jacobs, D. E. de Vos and J. Hofkens, Nature, 2006, 439, 572.

64 H. P. Lu, L. Xu and X. S. Xie, Science, 1998, 282, 1877.

65 K. Velonia, O. Flomenbom, D. Loos, S. Masuo, M. Cotlet, Y. Engelborghs, J. Hofkens, A. E. Rowan, J. Klafter, R. J. M. Nolte and F. C. De Schryver, Angew. Chem., Int. Ed., $2005,44,560$

66 B. J. McIntyre, M. B. Salmeron and G. A. Somorjai, Catal. Lett., 1992, 14, 263.

67 L. Ruan, F. Besenbacher, I. Stensgaard and E. Laegsgaard, Phys. Rev. Lett., 1992, 69, 3523.

68 F. M. Leibsle, P. W. Murray, S. M. Francis, G. Thornton and M. Bowker, Nature, 1993, 363, 706 .

69 X. Bao, J. V. Barth, G. Lehmpfuhl, R. Schuster, Y. Uchida, R. Schlögl and G. Ertl, Surf. Sci., 1993, 284, 14.

70 S.-W. Hla, L. Bartels, G. Meyer and K.-H. Rieder, Phys. Rev. Lett., 2000, 85, 2777.

71 L. Grill, M. Dyer, L. Lafferentz, M. Persson, M. V. Peters and S. Hecht, Nat. Nanotechnol., 2007, $2,687$.

72 N. A. A. Zwaneveld, R. Pawlak, M. Abel, D. Catalin, D. Gigmes, D. Bertin and L. Porte, J. Am. Chem. Soc., 2008, 130, 6678.

73 M. M. S. Abdel-Mottaleb, N. Schuurmans, S. De Feyter, J. van Esch, B. L. Feringa and F. C. De Schryver, Chem. Commun., 2002, 1894.

74 Y. Kikkawa, E. Koyama, S. Tsuzuki, K. Fujiwara, K. Miyake, H. Tokuhisa and M. Kanesato, Langmuir, 2002, 22, 6910.

75 P. C. M. Grim, S. De Feyter, A. Gesquière, P. Vanoppen, M. Rücker, S. Valiyaveettil, G. Moessner, K. Müllen and F. C. De Schryver, Angew. Chem., Int. Ed. Engl., 1997, 36, 2601.

76 Y. Okawa and M. Aono, Nature, 2001, 409, 683.

77 M. M. S. Abdel-Mottaleb, S. De Feyter, A. Gesquière, M. Sieffert, M. Klapper, K. Müllen and F. C. De Schryver, Nano Lett., 2001, 1, 353.

78 L. Y. O. Yang, C. Chang, S. Liu, C. Wu and S. L. Yau, J. Am. Chem. Soc., 2007, 129, 8076.

79 B. Hulsken, R. van Hameren, J. W. Gerritsen, T. Khoury, P. Thordarson, M. J. Crossley, A. E. Rowan, R. J. M. Nolte, J. A. A. W. Elemans and S. Speller, Nat. Nanotechnol., 2007, 2, 285. 